Sādhanā Vol. 40, Part 6, September 2015, pp. 1865-1898. (C) Indian Academy of Sciences

\title{
Vibration suppression during input tracking of a flexible manipulator using a hybrid controller
}

\author{
ASHISH SINGLA $^{1, *}$, ASHISH TEWARI ${ }^{2}$ and \\ BHASKAR DASGUPTA ${ }^{3}$
}
${ }^{1}$ Mechanical Engineering Department, Thapar University, Patiala 147004, India
${ }^{2}$ Department of Aerospace Engineering, Indian Institute of Technology Kanpur, Kanpur 208016, India
${ }^{3}$ Department of Mechanical Engineering, Indian Institute of Technology Kanpur, Kanpur 208016, India
e-mail: ashish.singla@thapar.edu

MS received 6 May 2014; revised 8 October 2014; accepted 7 April 2015

\begin{abstract}
The aim of this paper is to investigate the performance of the hybrid controller for end-point vibration suppression of a flexible manipulator, while it is tracking a desired input profile. Due to large structural vibrations, precise control of flexible manipulators is a challenging task. A hybrid controller is used to track large movements of flexible robotic manipulators, which is a combination of inverse dynamics feedforward control, command shaping and linear state feedback control. The case study of a single-link flexible manipulator is considered, where the manipulator is controlled under open-loop as well as closed-loop control scheme. In the open-loop control scheme, the aim is to test the effectiveness of the command shaper in reducing the vibration levels. Moreover, the effect of payload variations on the performance of command shapers and the importance of more robust shapers is demonstrated in this work. Under the closed-loop control scheme, the control objective is to track the large-hub angle trajectory, while maintaining low vibration levels. In comparison to collocated PD control, being reported in the literature, large reductions in tip acceleration levels as well as input torque magnitudes are observed with the proposed hybrid controller.
\end{abstract}

Keywords. Command shaping; vibration suppression; flexible links; finite element method; state estimation.

\section{Introduction}

Research in the field of flexible manipulators started in 1970s, when Book \& Majette (1983) initiated the research on modeling and control of the flexible link manipulators by modeling

*For correspondence 
an elastic chain with an arbitrary number of links and joints. The initial studies on the control of flexible manipulators started under the domain of space applications with the objective of minimizing the launching cost. Thereafter, flexible manipulators have been studied under various challenging applications like painting, drawing, polishing, pattern recognition, nuclear maintenance, storage tank cleaning and inspection, micro-surgical operation, automated deburring and many other similar operations. As compared with the conventional manipulators, flexible manipulators are lighter, faster, more portable, consume lesser power and possess high payloadto-robot-weight ratio. However, precise control of such manipulators is a challenging task, as flexible manipulators possess large structural vibrations.

To control flexible manipulators efficiently, the first and foremost step is to develop an accurate dynamic model of the system. In the past, various authors proposed different techniques to obtain the dynamic model of flexible manipulators. Tzou (1989) performed nonlinear finite-element analysis of flexible robotic manipulators with elastic joints using the pseudo-force approximation method. Usoro et al $(1984,1986)$ used the Lagrangian approach with finite element method, whereas De Luca \& Siciliano (1991) used the same approach with assumed modes method to obtain the mathematical model of lightweight flexible manipulators. Mohan \& Saha (2009) used decoupled natural orthogonal complement (DeNOC) matrices to formulate the dynamic equations of motion of a serial flexible-link manipulator. After developing an accurate mathematical model, the next step is to validate the dynamic model to use in simulation and control. The validation process is performed by comparing simulation and experimental results of the dynamic behavior of the flexible manipulator in two ways: (a) time-domain validation, which calculates time response of states of the system to an arbitrary input, and (b) frequency-domain validation, which calculates the parameters of the flexible system (natural frequencies and damping ratios).

Large movement tracking control of flexible manipulators along with vibration suppression require feedforward and feedback control schemes to be used in conjunction. Feedforward strategy includes Fourier expansion, computed torque techniques, bang-bang control (open-loop time-optimal control) and open-loop input shaping techniques. Out of these techniques, openloop input shaping (Singer \& Seering 1990) is used extensively by researchers as an active vibration suppression strategy in which the commanded signal is convolved with a sequence of impulses to obtain a non-oscillatory response. Open-loop input shaping is more sensitive to disturbances, which may arise due to unsuppressed higher modes, parametric uncertainties or inaccurate modeling of real-world systems. That is why, it is normally used in conjunction with feedback control schemes. Many attempts have been reported in the literature to handle the sensitivity problem using multiple modes (Hyde \& Seering 1991), robust shaping techniques (Singh \& Vadali 1994; Singhose et al 1996; Vaughan et al 2008), and combination of input-shaping with feedback control schemes (Drapeau \& Wang 1993; Hillsley \& Yurkovich 1993; Tewari 2004).

Under the feedback category, various control schemes like PD control (Yigit 1994), adaptive control (Feliu et al 1990; Shaheed \& Tokhi 2013), robust control (Rattan \& Feliu 1991), sliding mode control (Zhang et al 2004), feedback linearization control (Cambera et al 2014) have been implemented in the past on flexible manipulators. As compared to open-loop schemes, pure feedback schemes possess better robustness property, but require high control gains to suppress multiple modes. The major drawback of pure feedback controllers is the excitation of unmodeled modes which results in control spillover and large transient vibrations. It is possible to extract the benefits of open-loop and pure feedback control strategies while avoiding their individual drawbacks. The benefits of combining command shaping with feedback controller are demonstrated by Tzes \& Yurkovich (1993). Thereafter, several researchers in the past have applied input shaping to closed-loop systems with linear (Kapila et al 2000; Kenison \& Singhose 2002) as well 
as nonlinear feedback controllers (Banerjee \& Singhose 1998; Romano et al 2002; Zain et al 2006a).

To summarize, the authors can say that the state-of-the-art in large motion tracking of flexible manipulators employs efficient use of nonlinear feedback controllers. Also, linear feedback controllers are used in some references, but with non-optimal PD gains and using full-state feedback. In this paper, a closed-loop tracking system, also called as a hybrid controller, is used for vibration suppression of a single-link flexible manipulator undergoing large angle movements. The hybrid controller is designed by the combination of three different control schemes namely nonlinear feedforward control, linear feedback control and command shaping. The nonlinear feedforward control is obtained by dynamic inversion for generating the nominal trajectory to obtain linearized models. The linear feedback is designed with a linear quadratic regulator (LQR) which ensures stabilization as well as desired performance of the flexible systems. Further, a reduced order observer (ROO) is used to estimate unmeasurable states of a flexible manipulator and command shaping (CS) is utilized to reduce the vibration levels. With the help of simulation runs, the efficacy of the hybrid controller is demonstrated in terms of reductions in vibrations levels and input-torque magnitudes.

\section{Dynamic modeling of flexible manipulators}

This section presents the mathematical formulation to develop the dynamic model of flexible manipulators using finite element method (FEM). The finite element method regards a complex structure as an assemblage of finite number of elements treating each element as a continuous structural member (Meirovitch 1986). Further, the displacement at any point of the continuous element is represented in terms of finite number of nodal displacements multiplied by given interpolation functions.

\subsection{Finite element method}

Using finite element method, the partial differential equations of motion are transformed into finite number of second-order differential equations. Several finite element models for flexible manipulators have been presented by many researchers. In this work, small elastic deformations are assumed along with large rigid-body movements, in which small elastic displacements are measured relative to the large rigid-body motions, i.e. from the neutral axis of the link. Defining generalized coordinates of flexible modes in the inertial frame is not considered advisable, as it leads to nonlinear equations of motion for large movements of the manipulator. However, by defining such coordinates in a moving frame, equations of motion are greatly simplified (refer Junkins \& Kim 1993). It means that large rigid-body motions coupled with small elastic deformations can be effectively modeled by linear differential equations. The flexible link of the manipulator is considered to be made of finite number of elements of equal length. The elemental equations of motion are developed in Appendix.

\section{Case study: Single-link flexible manipulator}

This section presents the FEM model of a single-link flexible manipulator, as shown in figure 1. Euler-Bernoulli beam theory is used to model the elastic behavior of the manipulator and Lagrange approach is utilized to derive the equations of motion. Discretization has been done 


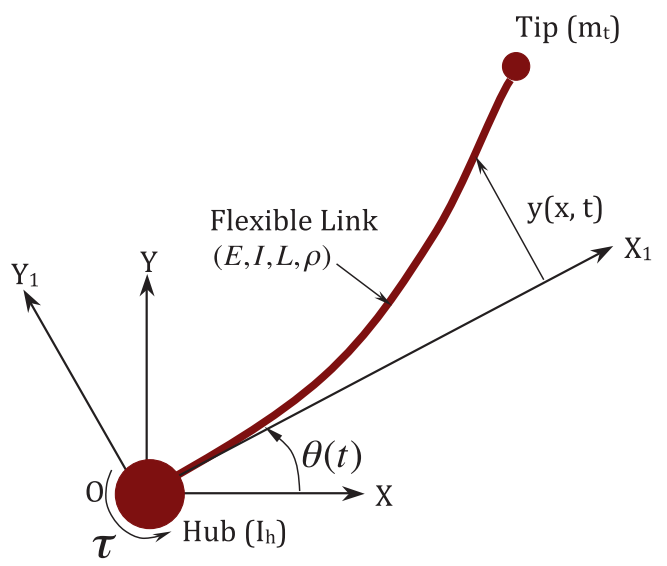

Figure 1. Schematic diagram of a single-link flexible manipulator.

through finite element approach. In the absence of deformation, each flexible link is assumed to be symmetrical about its longitudinal axis. The flexible link of the manipulator is considered to be rigid in compression, but not in bending. In this model, small elastic deformations are assumed along with large rigid-body movements, in which small elastic displacements are measured relative to the large rigid-body motions. The large rigid-body motions $(\theta)$ are provided by the actuator.

The various symbols used in the figure are described as

$$
\begin{aligned}
O X Y= & \text { inertial coordinate system, } \\
O_{1} X_{1} Y_{1}= & \text { body-fixed coordinate frame attached to the flexible link, } \\
\mathbf{r}= & \text { position vector of a point on the flexible link w.r.t. inertial }(O X Y) \text { frame, } \\
\mathbf{r}= & \text { position vector of a point on the flexible link link as measured w.r.t. } \\
& O_{1} X_{1} Y_{1} \text { frame, } \\
\theta= & \text { angular displacement of the joint, } \\
E= & \text { Young's modulus, } \\
I= & \text { moment of inertia, } \\
L= & \text { total length of flexible link, } \\
\rho= & \text { mass density (mass/length) of the flexible link, } \\
m_{t}= & \text { tip mass, } \\
I_{h}= & \text { hub inertia. }
\end{aligned}
$$

First of all, the kinetic and potential energy of each element is calculated to obtain the elemental mass and stiffness matrices. Thereafter, these elemental matrices are assembled suitably to obtain the global mass and stiffness matrices. The final nonlinear model is highly general as the desired accuracy can be obtained by suitably varying the number of elements per link. Actuator dynamics and structural damping is ignored here.

\subsection{Kinetic energy}

Kinetic energy of the whole flexible link is calculated by adding the elemental kinetic energy of each element of the flexible link. 
3.1a Elemental kinetic energy: The elemental kinetic energy i.e. $T_{j}$, for the $j$-th element is given as

$$
T_{j}=\frac{1}{2} \int_{0}^{l} \rho\left[\frac{\partial \mathbf{r}}{\partial t}\right]^{T} \frac{\partial \mathbf{r}}{\partial t} d x_{j} .
$$

For convenience, $\mathbf{r}$ can be written, in terms of $\mathbf{r}_{1}$ w.r.t. $O_{1} X_{1} Y_{1}$ frame, as

$$
\mathbf{r}=\mathbf{T}_{0}^{1} \mathbf{r}_{1}
$$

where

$$
\begin{aligned}
\mathbf{r}_{1} & =\left[\begin{array}{c}
(j-1) l+x_{j} \\
y_{j}
\end{array}\right], \\
\mathbf{T}_{0}^{1} & =\left[\begin{array}{cc}
\cos (\theta) & -\sin (\theta) \\
\sin (\theta) & \cos (\theta)
\end{array}\right] .
\end{aligned}
$$

The displacement $y_{j}$ can be represented in terms of shape functions $\phi_{k}(x)$ and nodal displacements $u(t)$ as

$$
y_{j}\left(x_{j}, t\right)=\sum_{k=1}^{4} \phi_{k}\left(x_{j}\right) u_{2 j-2+k}(t) .
$$

As the model is based on Euler-Bernoulli beam theory, the finite element method needs each node to possess two degrees of freedom, a transverse deflection and rotation. This motivates the use of Hermite cubics as the elemental shape functions (Meirovitch 1986).

Finally, using Eqs. (1) to (5), elemental kinetic energy (Tokhi et al 2001) of the flexible link can be given as

$$
\begin{aligned}
T_{j} & =\frac{1}{2} \int_{0}^{l} \rho\left[\frac{\partial \mathbf{r}}{\partial t}\right]^{T} \frac{\partial \mathbf{r}}{\partial t} d x_{j} \\
& =\frac{1}{2} \dot{\mathbf{z}}_{j}^{T} \mathbf{M}_{j} \dot{\mathbf{z}}_{j}
\end{aligned}
$$

where

$$
\begin{aligned}
& \mathbf{z}_{j}=\left[\begin{array}{lllll}
\theta & u_{2 j-1} & u_{2 j} & u_{2 j+1} & u_{2 j+2}
\end{array}\right]^{T}, \\
& \mathbf{M}_{j}=\int_{0}^{l} \rho\left[\frac{\partial \mathbf{r}}{\partial \mathbf{z}_{j}}\right]^{T} \frac{\partial \mathbf{r}}{\partial \mathbf{z}_{j}} d x_{j} .
\end{aligned}
$$

As $\mathbf{z}_{j}$ contains 5 elements, so $\mathbf{M}_{j}$ will be a $5 \times 5$ matrix with the following structure

$$
\mathbf{M}_{j}=\left[\begin{array}{cccc}
m_{j}(1,1) & m_{j}(1,2) & \ldots & m_{j}(1,5) \\
m_{j}(1,2) & & & \\
\vdots & & \mathbf{P}_{j} &
\end{array}\right]
$$


where

$$
\begin{aligned}
m_{j}(1,1) & =\frac{\rho l^{3}}{3}\left(3 j^{2}-3 j+1\right)+\psi_{j}^{T} \mathbf{P}_{j} \psi_{j}, \\
m_{j}(1,2) & =\frac{\rho l^{2}}{20}(10 j-7), \\
m_{j}(1,3) & =\frac{\rho l^{3}}{60}(5 j-3), \\
m_{j}(1,4) & =\frac{\rho l^{2}}{20}(10 j-3), \\
m_{j}(1,5) & =-\frac{\rho l^{3}}{60}(5 j-2),
\end{aligned}
$$

and

$$
\begin{aligned}
& \psi_{j}=\left[\begin{array}{llll}
u_{2 j-1} & u_{2 j} & u_{2 j+1} & u_{2 j+2}
\end{array}\right]^{T}, \\
& \mathbf{P}_{j}=\mathbf{M}^{e} .
\end{aligned}
$$

In the above expression, $\mathbf{P}_{j}$ is equal to the elemental mass matrix of the beam as given by Eq. (85).

3.1b Total kinetic energy of the first link: Total kinetic energy of the first link can be calculated by combining the elemental kinetic energies of all the $n$ elements as

$$
\bar{T}=\sum_{j=1}^{n} T_{j}=\frac{1}{2} \dot{\overline{\mathbf{q}}}^{T} \overline{\mathbf{M}} \dot{\overline{\mathbf{q}}},
$$

where

$$
\begin{aligned}
& \overline{\mathbf{q}}=\left[\theta, \bar{\psi}^{T}\right]^{T},
\end{aligned}
$$

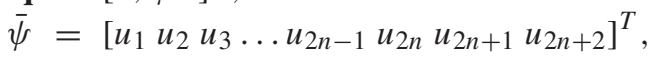

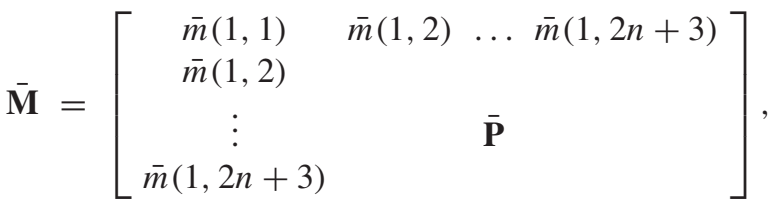

$$
\begin{aligned}
& \overline{\mathbf{P}}=\overline{\mathbf{M}}^{g} \text {. }
\end{aligned}
$$

In the above expression, $\overline{\mathbf{P}}$ is calculated by using Eq. (91). The elements of $\overline{\mathbf{M}}$ are given as

$$
\begin{aligned}
& \bar{m}(1,1)=\frac{\rho l^{3} n^{3}}{3}+\bar{\psi}^{T} \overline{\mathbf{P}} \bar{\psi}, \\
& \bar{m}(1,2)=\frac{3 \rho l^{2}}{20}, \\
& \bar{m}(1,3)=\frac{\rho l^{3}}{30}, \\
& \bar{m}(1,4)=\rho l^{2},
\end{aligned}
$$




$$
\begin{array}{rlrl}
\bar{m}(1,5) & =\frac{\rho l^{3}}{15}, & & \\
\bar{m}(1,2 k) & =(k-1) \rho l^{2}, & & \\
\bar{m}(1,2 k+1) & =\frac{\rho l^{3}}{15}, & & \\
\bar{m}(1,2 n+2) & =\frac{\rho l^{2}}{20}(10 n-3), & & \\
\bar{m}(1,2 n+3) & =-\frac{\rho l^{3}}{60}(5 n-2) ., 3, \ldots, n
\end{array}
$$

3.1c Boundary conditions: After applying the boundary conditions i.e. $u_{1}=u_{2}=0$ by appropriately deleting the rows and columns of $\overline{\mathbf{M}}$, the total kinetic energy of the flexible link can be given as

$$
T=\frac{1}{2} \dot{\mathbf{q}}^{T} \mathbf{M} \dot{\mathbf{q}}
$$

where

$$
\begin{aligned}
& \mathbf{q}=\left[\theta, \psi^{T}\right]^{T}, \\
& \psi=\left[\begin{array}{llllll}
u_{3} & \ldots & u_{2 n-1} & u_{2 n} & u_{2 n+1} & u_{2 n+2}
\end{array}\right]^{T},
\end{aligned}
$$

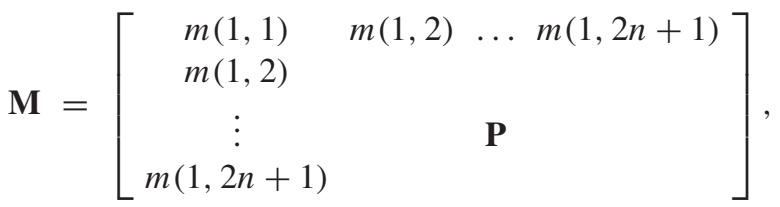

$$
\begin{aligned}
& \mathbf{P}=\mathbf{M}^{g} \text {. }
\end{aligned}
$$

Here, $\mathbf{P}$ is calculated using Eq. (96). The elements of generalized inertia matrix $\mathbf{M}$ are given as

$$
\begin{array}{rlrl}
m(1,1) & =\frac{\rho l^{3} n^{3}}{3}+\psi^{T} \mathbf{P} \psi, & & \\
m(1,2 k-2) & =(k-1) \rho l^{2}, & \forall & k=2,3, \ldots, n \\
m(1,2 k-1) & =\frac{\rho l^{3}}{15}, & & \\
m(1,2 n) & =\frac{\rho l^{2}}{20}(10 n-3), & & \\
m(1,2 n+1) & =-\frac{\rho l^{3}}{60}(5 n-2) ., \ldots, n
\end{array}
$$

\subsection{Potential energy}

Potential energy of the flexible link is calculated by adding the elemental potential energy of each element.

3.2a Elemental potential energy: The elemental potential energy i.e. $V_{j}$, for the $j^{\text {th }}$ element of the flexible link is given as

$$
V_{j}=V_{g_{j}}+V_{e_{j}},
$$


where

$$
\begin{aligned}
& V_{g_{j}}=\text { Gravitational potential energy }=\int_{0}^{l} \rho g[01] \mathbf{r} d x, \\
& V_{e_{j}}=\text { Elastic potential energy }=\frac{1}{2} \int_{0}^{l} E I\left[\frac{d^{2} y_{j}}{d x^{2}}\right]^{2} d x,
\end{aligned}
$$

Thus, the elemental potential energy is given as

$$
\begin{aligned}
& V_{j}=\int_{0}^{l} \rho g\left[\begin{array}{ll}
0 & 1
\end{array}\right] \mathbf{r} d x+\frac{1}{2} \int_{0}^{l} E I\left[\frac{d^{2} y_{j}}{d x^{2}}\right]^{2} d x, \\
& =\int_{0}^{l} \rho g\left[\begin{array}{ll}
0 & 1
\end{array}\right] \mathbf{T}_{0}^{1}\left[\begin{array}{c}
(j-1) l+x \\
y
\end{array}\right] d x+\frac{1}{2} \int_{0}^{l} E I\left[\frac{d^{2} y_{j}}{d x^{2}}\right]^{2} d x, \\
& =\rho g\left[\begin{array}{ll}
0 & 1
\end{array}\right] \mathbf{T}_{0}^{1}\left[\begin{array}{c}
\left(j-\frac{1}{2}\right) l^{2} \\
\frac{l}{2} u_{2 j-1}+\frac{l^{2}}{12} u_{2 j}+\frac{l}{2} u_{2 j+1}-\frac{l^{2}}{12} u_{2 j+2}
\end{array}\right] \\
& +\frac{1}{2}\left[\begin{array}{llll}
u_{2 j-1} & u_{2 j} & u_{2 j+1} & u_{2 j+2}
\end{array}\right] \mathbf{K}_{j}\left[\begin{array}{c}
u_{2 j-1} \\
u_{2 j} \\
u_{2 j+1} \\
u_{2 j+2}
\end{array}\right] \text {, }
\end{aligned}
$$

where

$$
\mathbf{K}_{j}=\mathbf{K}^{e} .
$$

In the above expression, $\mathbf{K}_{j}$ is equal to the elemental stiffness matrix of the beam and is calculated using Eq. (88). Further, total potential energy of the flexible link is calculated by adding all the elemental potential energies.

$$
\bar{V}=\bar{V}_{g}+\bar{V}_{e}
$$

where

$$
\begin{aligned}
\bar{V}_{g} & =\rho g\left[\begin{array}{ll}
0 & 1
\end{array}\right] \mathbf{T}_{0}^{1}\left[\begin{array}{c}
\frac{1}{2} n l^{2} \\
\overline{\mathbf{R}} \bar{\psi}
\end{array}\right], \\
\bar{V}_{e} & =\frac{1}{2} \bar{\psi}^{T} \overline{\mathbf{K}} \bar{\psi}, \\
\overline{\mathbf{R}} & \left.=\left[\begin{array}{llllll}
\frac{l}{2} & \frac{l^{2}}{12} \mid l l & 0 \mid l l & 0
\end{array}\right] \ldots|l \quad 0| \frac{l}{2}-\frac{l^{2}}{12}\right], \quad \forall i=1,2, \\
\overline{\mathbf{K}} & =\overline{\mathbf{K}}^{g} .
\end{aligned}
$$


In the above expression, $\overline{\mathbf{K}}$ is calculated by using Eq. (93). Incorporating the boundary conditions i.e. $u_{1}=u_{2}=0$ by deleting the rows and columns appropriately, the total potential energy of link 1 can be found as

$$
\begin{aligned}
V & =V_{g}+V_{e} \\
& =\rho g\left[\begin{array}{ll}
0 & 1
\end{array}\right] \mathbf{T}_{0}^{1}\left[\begin{array}{c}
\frac{1}{2} n l^{2} \\
\mathbf{R} \psi
\end{array}\right]+\frac{1}{2} \psi^{T} \mathbf{K} \psi, \\
\mathbf{R} & =\left[\begin{array}{lllllll}
l & 0 & l & 0 & \ldots
\end{array}\right] \\
\mathbf{K} & =\mathbf{K}^{g},
\end{aligned}
$$

In the above expression, $\mathbf{K}$ is calculated by calculated by using Eq. (98).

\subsection{Dynamic model of a single-link flexible manipulator}

To calculate the dynamic model of the system, Lagrange's equations are used as given below

$$
\frac{d}{d t}\left[\frac{\partial \mathcal{L}}{\partial \dot{\mathrm{q}}}\right]-\left[\frac{\partial \mathcal{L}}{\partial \mathrm{q}}\right]=\tau,
$$

where $\mathcal{L}$ is the Lagrangian of the system, $\mathbf{q}$ is the vector of generalized coordinates and $\tau$ is the vector of generalized nonconservative forces.

The Lagrangian $\mathcal{L}$ for the flexible link is given as

$$
\mathcal{L}\left(\theta, \psi^{T}\right)=T-V
$$

where $\psi, T$, and $V$ are given by Eqs. (21), (19), and (35).

By using Lagrange's equations as given by Eq. (38), dynamic model of the single-link flexible manipulator is written as

$$
\mathbf{M}(\mathbf{q}) \ddot{\mathbf{q}}+\mathbf{n}(\mathbf{q}, \dot{\mathbf{q}})=\tau,
$$

where

$$
\begin{aligned}
\mathbf{q} & =\left[\theta, \psi^{T}\right]^{T}=\text { vector of generalized coordinates, } \\
\tau & =\left[\tau, \mathbf{f}^{T}\right]^{T}=\text { vector of generalized nonconservative forces, } \\
\mathbf{f} & =\left[f_{13}, f_{14}, \ldots, f_{1,2 n+2}\right]^{T}=\text { nodal force vector of the link, } \\
\mathbf{M}(\mathbf{q}) & =\mathbb{R}^{\left(2 n+2 n_{2}+2\right) \times\left(2 n+2 n_{2}+2\right)} \text { symmetric positive-definite inertia matrix, } \\
\mathbf{n}(\mathbf{q}, \dot{\mathbf{q}}) & =\text { vector of Coriolis, centripetal, gravitational and elastic forces, } \\
& =-\left[\frac{\partial \mathcal{L}}{\partial \theta}, \frac{\partial \mathcal{L}}{\partial u_{3}}, \ldots, \frac{\partial \mathcal{L}}{\partial u_{2 n}}, \frac{\partial \mathcal{L}}{\partial u_{2 n+1}}, \frac{\partial \mathcal{L}}{\partial u_{2 n+2}}\right]^{T},
\end{aligned}
$$


with

$$
\begin{aligned}
\frac{\partial \mathcal{L}}{\partial \dot{\mathbf{q}}} & =\mathbf{M} \dot{\mathbf{q}}, \\
\frac{\partial \mathcal{L}}{\partial \theta} & =-\rho g\left[\begin{array}{ll}
1 & 0
\end{array}\right] \mathbf{T}_{0}^{1}\left[\begin{array}{c}
\frac{1}{2} n^{2} l^{2} \\
\mathbf{R} \psi
\end{array}\right], \\
\frac{\partial \mathcal{L}}{\partial \psi} & =\dot{\theta}^{2} \mathbf{P} \psi-\rho g\left[\mathbf{0} \mathbf{R}^{T}\right] \mathbf{T}_{0}^{1^{T}}\left[\begin{array}{l}
0 \\
1
\end{array}\right]-\mathbf{K} \psi,
\end{aligned}
$$

\subsection{State space representation}

By writing the state vector as $\mathbf{x}(t)=\left\{\tilde{\mathbf{q}}(t)^{T}, \dot{\tilde{\mathbf{q}}}(t)^{T}\right\}^{T}$ with $\tilde{\mathbf{q}}(t)=\theta(t)-\theta_{n}$ as the deviation from the nominal trajectory, the dynamic model in (40) is linearized about the nominal trajectory and can be written in the state-space form as

$$
\dot{\mathbf{x}}(t)=\mathbf{A x}(t)+\mathbf{B u}(t) .
$$

where $\mathbf{A}$ and $\mathbf{B}$ are the following Jacobian matrices of the system linearized about the nominal trajectory with $\boldsymbol{P}\left(\boldsymbol{\tau}_{\boldsymbol{n}}, \boldsymbol{\theta}_{\boldsymbol{n}}, \dot{\boldsymbol{\theta}}_{\boldsymbol{n}}, \ddot{\boldsymbol{\theta}}_{\boldsymbol{n}}\right)$ as the nominal point.

$$
\mathbf{A}=\left[\begin{array}{cc}
\mathbf{0}_{n} & \mathbf{I}_{n} \\
-\mathbf{M}_{L}^{-1} \mathbf{G}_{L} & -\mathbf{M}_{L}^{-1} \mathbf{N}_{L}
\end{array}\right], \quad \mathbf{B}=\left\{\begin{array}{c}
\mathbf{0}_{n} \\
\mathbf{M}_{L}^{-1}
\end{array}\right\} .
$$

where

$$
\begin{aligned}
\mathbf{M}_{L} & =[\mathbf{M}(\theta)]_{P}=n \times n \text { matrix of inertia forces, } \\
\mathbf{N}_{L} & =\left[\frac{\partial \mathbf{n}}{\partial \dot{\theta}}\right]_{P}=n \times n \text { matrix of Coriolis and centripetal forces, } \\
\mathbf{G}_{L} & =\left[\frac{\partial(\mathbf{n})}{\partial \theta}\right]_{P}=n \times n \text { matrix of gravitational forces. }
\end{aligned}
$$

This state-space representation of the dynamic model is helpful in checking the controllability. Moreover, it helps in designing the feedback loop of the controller, which is based on the linear control theory.

\section{Validation of the dynamic model}

To obtain realistic results, the parameters of the manipulator are kept same as used by Tokhi et al (2001), and are given in table 1. The authors neglected the transverse shear and rotary inertia effects, as the manipulator link is considered to be long and slender. The link with constant cross-section and uniform material properties, is assumed to be stiff in vertical direction. Gravitational and dynamic stiffening effects are also neglected.

Before going to the control phase, the most important step is to validate the nonlinear dynamic model. The model is validated at two stages - frequency-domain validation and time-domain validation. The former deals with the verification of natural frequencies of the system, which has a direct bearing on the accuracy of the dynamic model, whereas the latter deals with the transient as well as steady-state response of the system to a given input. In the frequency-domain 
Table 1. System parameters of a single-link flexible manipulator.

\begin{tabular}{lccc}
\hline Parameter & Units & Symbol & Value \\
\hline Link length & $\mathrm{m}$ & $L$ & 0.9 \\
Link width & $\mathrm{m}$ & $b$ & $1.9008 \times 10^{-2}$ \\
Link height & $\mathrm{m}$ & $h$ & $3.2004 \times 10^{-3}$ \\
Cross sectional area & $\mathrm{m}^{2}$ & $A$ & $6.0833 \times 10^{-5}$ \\
Mass density per unit volume & $\mathrm{kg} / \mathrm{m}^{3}$ & $\rho$ & 2710 \\
Area moment of inertia & $\mathrm{m}^{4}$ & $I$ & $5.1924 \times 10^{-11}$ \\
Hub inertia & $\mathrm{kgm}^{2}$ & $I_{h}$ & $5.8598 \times 10^{-4}$ \\
Young's modulus & $\mathrm{N} / \mathrm{m}^{2}$ & $E$ & $71 \times 10^{9}$ \\
\hline
\end{tabular}

validation, all the resonant frequencies are obtained by using finite-element formulation written in Matlab. Table 2 shows the comparison of first three resonant frequencies, as a function of number of elements, with the corresponding values reported in Tokhi et al (2001). The table shows the resonant frequencies to be in close agreement. Further, figure 2 shows the time-response of the single-link flexible manipulator using a single element to a bang-bang torque profile (shown in figure 4). Figure 2 shows the response of end-point displacement, end-point acceleration, hubangle position and hub-angle velocity, which are found to be in close agreement with Tokhi et al results. Both the steps reported above completely validates the model of the manipulator.

\section{Controller design}

In this section, the procedure for controller design for the tracking problem of nonlinear, unstable flexible manipulators is developed. The control action ( $\mathbf{u})$ of the considered controller is given as

$$
\mathbf{u}=\mathbf{u}_{f f}+\mathbf{u}_{f b},
$$

As shown in figure 3, the controller consists of three parts: (1) a nonlinear feedforward term $\left(\mathbf{u}_{f f}\right),(2)$ a linear observer based optimal feedback term $\left(\mathbf{u}_{f b}\right)$ and (3) an input shaper. Let us discuss each term in detail.

\subsection{Nonlinear feedforward control}

Feedforward control is very useful at places where the robot has to perform fast and repetitive moves. The use of feedforward control provides an efficient solution to this problem as the whole

Table 2. Model validation: Comparison of first three resonant frequencies.

\begin{tabular}{|c|c|c|c|c|c|c|}
\hline \multirow{2}{*}{$\begin{array}{l}\text { Number of } \\
\text { elements } \\
(n)\end{array}$} & \multicolumn{3}{|c|}{$\begin{array}{l}\text { Resonant frequencies in } \mathrm{Hz} \\
\text { reported by Tokhi et al (2001) }\end{array}$} & \multicolumn{3}{|c|}{$\begin{array}{l}\text { Resonant frequencies in } \mathrm{Hz} \\
\text { calculated with FEM code }\end{array}$} \\
\hline & $\overline{\text { Mode } 1}$ & Mode 2 & Mode 3 & $\overline{\text { Mode } 1}$ & Mode 2 & Mode 3 \\
\hline 1 & 14.49 & 47.70 & - & 14.36 & 47.45 & - \\
\hline 2 & 11.99 & 35.71 & 77.17 & 13.37 & 35.55 & 76.69 \\
\hline 5 & 11.99 & 35.46 & 65.43 & 13.31 & 35.15 & 65.04 \\
\hline 10 & 11.99 & 35.22 & 65.20 & 13.31 & 35.11 & 64.83 \\
\hline 20 & 11.99 & 35.22 & 65.20 & 13.31 & 35.11 & 64.83 \\
\hline
\end{tabular}




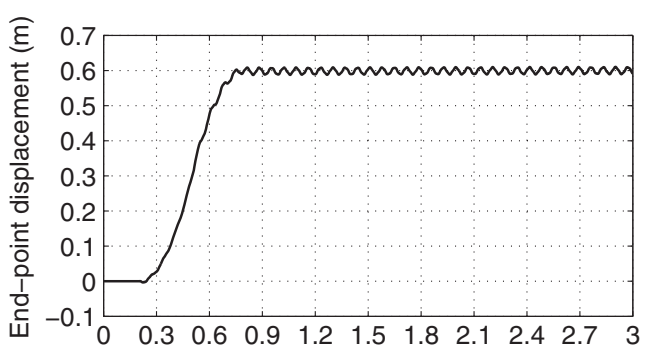

(a)

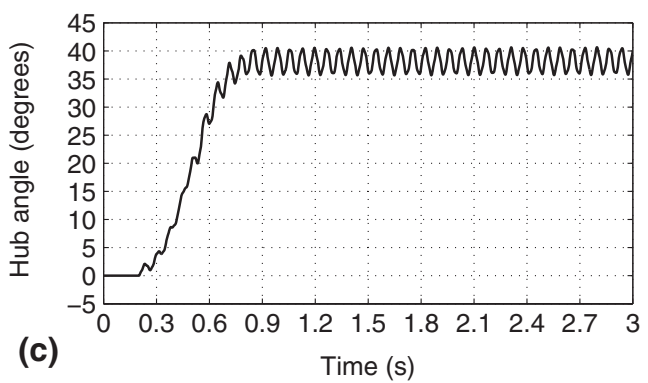

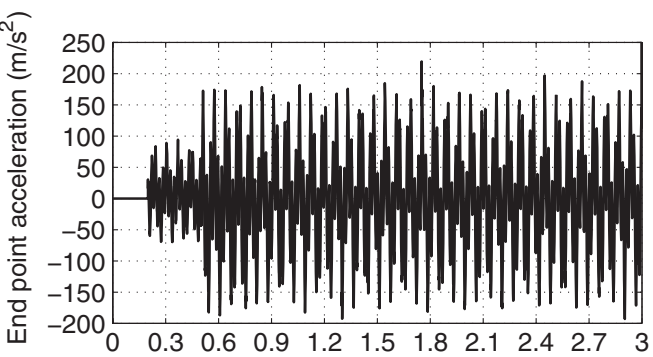

(b)

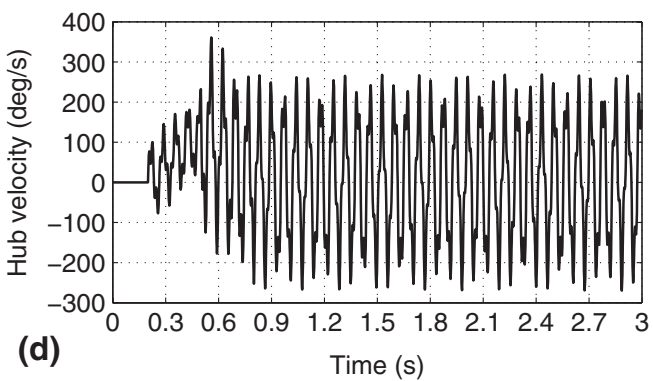

Figure 2. Model validation: Bang-bang response of a single-link flexible manipulator using one element.

computation is done off-line along the nominal trajectory. Given the desired positions $\left(\theta_{d}(t)\right)$, velocities $\left(\dot{\theta}_{d}(t)\right)$ and accelerations $\left(\ddot{\theta}_{d}(t)\right)$ in joint space, joint torques are calculated by the dynamic inversion. The feedforward control action $\left(\mathbf{u}_{f f}\right)$ is given as

$$
\mathbf{u}_{f f}=\mathbf{M}\left(\theta_{d}\right) \ddot{\theta}_{d}+\mathbf{n}\left(\theta_{d}, \dot{\theta}_{d}\right) .
$$

It can be seen from the above equation that feedforward control is nonlinear and comes under the category of open-loop control as it does not depend on $\mathbf{q}$ and $\dot{\mathbf{q}}$.

\subsection{Linear feedback control}

In this section, the compensator design is presented, which is based on reduced-order observer as all the states of flexible manipulator are not measurable. The state-vector $\mathbf{x}(t)$ of the

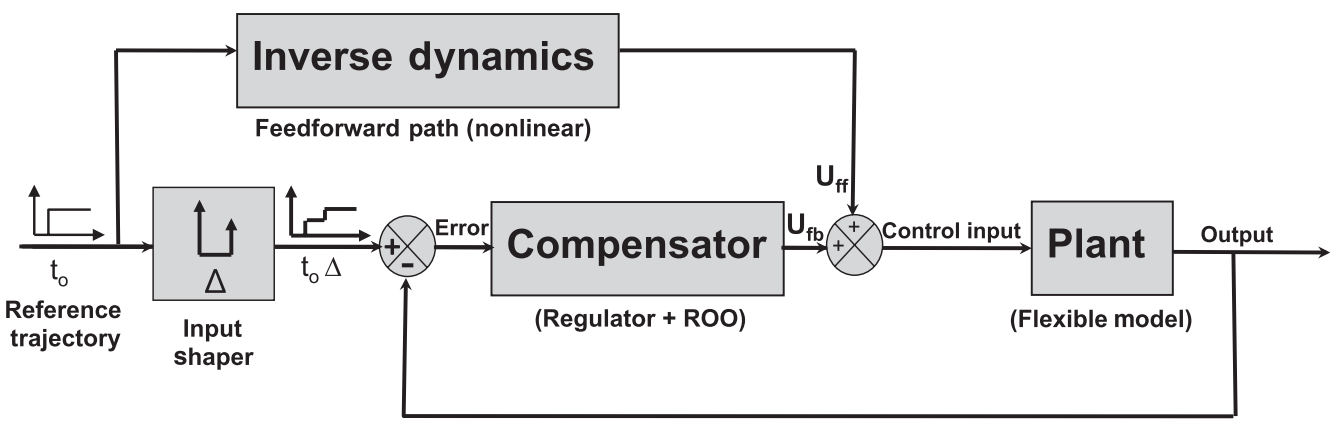

Feedback loop (linear)

Figure 3. Schematic diagram of the proposed tracking controller. 
flexible manipulator can be partitioned as $\mathbf{x}(t)=\left[\mathbf{x}_{m}(t)^{T}, \mathbf{x}_{u}(t)^{T}\right]^{T}$, where $\mathbf{x}_{m}(t)$ is the vector of measured state variables and $\mathbf{x}_{u}(t)$ is the vector of unmeasured state variables. The vector of measured outputs i.e. $\mathbf{y}(t)$ can directly be chosen as equal to $\mathbf{x}_{m}(t)$ or any linear combination of all the state variables of $\mathbf{x}_{m}(t)$. The output equation is given as

$$
\mathbf{y}(t)=\mathbf{C} \mathbf{x}_{m}(t)
$$

where $\mathbf{C}$ is a constant, square matrix, indicating the same number of outputs as the dimension of $\mathbf{x}_{m}(t)$. The choice of $\mathbf{C}=\mathbf{I}$ means that all the elements of $\mathbf{x}_{m}(t)$ are directly measurable. The state-equation (49) of the flexible manipulator can be expressed in partitioned form as

$$
\begin{aligned}
\dot{\mathbf{x}}_{m}(t) & =\mathbf{A}_{m m} \mathbf{x}_{m}(t)+\mathbf{A}_{m u} \mathbf{x}_{u}(t)+\mathbf{B}_{m} \mathbf{u}(t), \\
\dot{\mathbf{x}}_{u}(t) & =\mathbf{A}_{u m} \mathbf{x}_{m}(t)+\mathbf{A}_{u u} \mathbf{x}_{u}(t)+\mathbf{B}_{u} \mathbf{u}(t),
\end{aligned}
$$

where the state coefficient matrices in partitioned form can be expressed as

$$
\mathbf{A}=\left[\begin{array}{cc}
\mathbf{A}_{m m} & \mathbf{A}_{m u} \\
\mathbf{A}_{u m} & \mathbf{A}_{u u}
\end{array}\right], \quad \mathbf{B}=\left[\begin{array}{c}
\mathbf{B}_{m} \\
\mathbf{B}_{u}
\end{array}\right] .
$$

With the order of the plant be $n$ and the number of measures state variables be $k$, a reducedorder observer of dimension $(n-k)$ is required to estimate all the elements of $\mathbf{x}_{u}(t)$. The estimated state vector $\mathbf{x}_{o}(t)$ can be written as

$$
\mathbf{x}_{o}(t)=\left[\begin{array}{c}
\mathbf{x}_{m}(t) \\
\mathbf{x}_{o u}
\end{array}\right]=\left[\begin{array}{c}
\mathbf{C}^{-1} \mathbf{y}(t) \\
\mathbf{x}_{o u}
\end{array}\right],
$$

where $\mathbf{x}_{o u}(t)$ is the estimation of the unmeasurable state-vector $\mathbf{x}_{u}(t)$. To design a reduced-order observer, $\mathbf{C}$ should be invertible.

\subsection{Reduced-order observer design}

The objective of the observer is to achieve zero estimation error in the steady state. The estimation error $\mathbf{e}_{o u}(t)$ can be expressed as

$$
\mathbf{e}_{o u}(t)=\mathbf{x}_{u}(t)-\mathbf{x}_{o u}(t) .
$$

The reduced-order observer dynamics is given by

$$
\mathbf{x}_{\text {ou }}(t)=\mathbf{L y}(t)+\mathbf{z}(t),
$$

where $\mathbf{L}$ is the observer gain matrix and $\mathbf{z}(t)$ is the solution of the following state equation

$$
\dot{\mathbf{z}}(t)=\mathbf{F z}(t)+\mathbf{H u}(t)+\mathbf{G y}(t) .
$$

The state equation for estimation error (refer Tewari 2002) can be given as

$$
\begin{aligned}
\dot{\mathbf{e}}_{o u}(t) & =\dot{\mathbf{x}}_{u}(t)-\dot{\mathbf{x}}_{o u}(t) \\
& =\mathbf{F e}_{o u}(t)+\left(\mathbf{A}_{u m}-\mathbf{L C A} \mathbf{A}_{m m}+\mathbf{F L C}-\mathbf{G C}\right) \mathbf{x}_{m}(t)+\ldots \\
& +\left(\mathbf{A}_{u u}-\mathbf{L C A} \mathbf{A}_{m u}-\mathbf{F}\right) \mathbf{x}_{u}(t)+\left(\mathbf{B}_{u}-\mathbf{L C B} \mathbf{B}_{m}-\mathbf{H}\right) \mathbf{u}(t) .
\end{aligned}
$$

To achieve zero estimation error at the steady state, all the coefficient matrices of $\mathbf{x}_{m}(t), \mathbf{x}_{u}(t)$ and $\mathbf{u}(t)$ must vanish and all the eigenvalues of $\mathbf{F}$ must lie in the left-half plane. This leads to

$$
\begin{aligned}
\mathbf{F} & =\mathbf{A}_{u u}-\mathbf{L C A}_{m u}, \\
\mathbf{H} & =\mathbf{B}_{u}-\mathbf{L C} \mathbf{C B}_{m}, \\
\mathbf{G} & =\mathbf{F L}+\left(\mathbf{A}_{u u}-\mathbf{L C} \mathbf{A}_{m m}\right) \mathbf{C}^{-1} .
\end{aligned}
$$


The reduced-order observer design is based on the selection of observer gain matrix $\mathbf{L}$, such that all the eigenvalues of $\mathbf{F}$ must lie in the left-half plane.

\subsection{Optimal feedback control}

The feedback control law based on the reduced-order observer for a tracking problem can be written as

$$
\begin{aligned}
\mathbf{u}_{f b}(t) & =\mathbf{K}_{o p t}\left(\mathbf{x}_{d}(t)-\mathbf{x}_{o}(t)\right) \\
& =\mathbf{K}_{o p t} \mathbf{x}_{d}(t)-\mathbf{K}_{m} \mathbf{x}_{m}(t)-\mathbf{K}_{u} \mathbf{x}_{o u}(t),
\end{aligned}
$$

where $\mathbf{x}_{d}(t)=\left\{\mathbf{q}_{d}^{T}, \dot{\mathbf{q}}_{d}^{T}\right\}^{T}$ is the desired state-vector and $\mathbf{K}_{\text {opt }}$ is the optimal feedback gain matrix being partitioned as $\mathbf{K}_{o p t}=\left[\mathbf{K}_{m} \mathbf{K}_{u}\right]$.

The feedback gain matrix $\mathbf{K}_{\text {opt }}$ is obtained by exploiting the optimal control theory, which produces the best possible control system to achieve the desired performance by formulating the following objective function

$$
J_{\infty}(t)=\int_{t}^{\infty}\left[\mathbf{x}^{T}(\tau) \mathbf{Q}(\tau) \mathbf{x}(\tau)+\mathbf{u}^{T}(\tau) \mathbf{R}(\tau) \mathbf{u}(\tau)\right] d(\tau),
$$

which is subjected to the following constraint

$$
\dot{\mathbf{x}}(t)=\mathbf{A}(t) \mathbf{x}(t)+\mathbf{B}(t) \mathbf{u}(t) .
$$

The above formulation is called as a constrained optimization problem in which $J_{\infty}(t)$ defines the objective function of the optimal control problem at steady-state. The scalar function $\mathbf{u}^{T}(t) \mathbf{R}(t) \mathbf{u}(t)$ represents control energy, where $\mathbf{R}(t)$ is a square, symmetric and positive definite control cost matrix. Similarly, the term $\mathbf{x}^{T}(t) \mathbf{Q}(t) \mathbf{x}(t)$ represents transient energy, where $\mathbf{Q}(t)$ is a square, symmetric and positive semi-definite state weighing matrix. The outcome of the optimal control problem is the feedback gain matrix $\mathbf{K}_{\text {opt }}$ such that the scalar function $J_{\infty}(t)$ is minimized. This minimization is performed in such a way that the state-vector $\mathbf{x}(t)$ is the solution to state-equation of the plant, as given in (70).

Finally, the error dynamics of the reduced-order compensator (Tewari 2002) can be written as

$$
\left\{\begin{array}{c}
\dot{\mathbf{e}}(t) \\
\dot{\mathbf{e}}_{o u}(t)
\end{array}\right\}=\left[\begin{array}{cc}
\mathbf{A}-\mathbf{B K}_{o p t} & -\mathbf{B} \mathbf{K}_{u} \\
\mathbf{0} & \mathbf{F}
\end{array}\right]\left\{\begin{array}{c}
\mathbf{e}(t) \\
\mathbf{e}_{o u}(t)
\end{array}\right\}+\left\{\begin{array}{c}
-\mathbf{A} \\
\mathbf{0}
\end{array}\right\} \mathbf{x}_{d}(t),
$$

where $\mathbf{e}(t)=\mathbf{x}_{d}(t)-\mathbf{x}(t)$ is the tracking error and $\mathbf{e}_{o u}(t)=\mathbf{x}_{u}(t)-\mathbf{x}_{o u}(t)$ is the estimation error. The above state space form of the complete dynamic system can be solved using Runge-Kutta 4(5) method with adaptive step size.

\subsection{Command shaping}

The method involves the convolution of the reference input with a sequence of impulses to obtain a non-oscillatory response, as shown in figure 3 . The $\Delta$ represents the time by which the second impulse is delayed from the first one. The input shaper variables, i.e. amplitudes $\left(A_{i}\right)$ and time locations $\left(t_{i}\right)$ of the impulses are found by satisfying a set of constraint equations which are functions of natural frequencies and damping ratios of the flexible system. However, the commanded signal comes with a marginal time penalty equal to the length of the shaper. 
In order to calculate the shaper variables, it is required to calculate the percentage residual vibration (PRV) (Vaughan et al 2008), which can be written as

$$
\begin{gathered}
V\left(\omega_{n}, \zeta\right)=e^{-\zeta \omega_{n} t_{N}} \sqrt{\left[V_{C}\left(\omega_{n}, \zeta\right)\right]^{2}+\left[V_{S}\left(\omega_{n}, \zeta\right)\right]^{2}} \\
V_{C}\left(\omega_{n}, \zeta\right)=\sum_{i=1}^{N} A_{i} e^{\zeta \omega_{n} t_{i}} \cos \left(\omega_{d} t_{i}\right) \\
V_{S}\left(\omega_{n}, \zeta\right)=\sum_{i=1}^{N} A_{i} e^{\zeta \omega_{n} t_{i}} \sin \left(\omega_{d} t_{i}\right)
\end{gathered}
$$

5.5a Zero vibration (ZV) shaper: With the sensible use of two impulses, vibration-free response of the system can be obtained. These, first shapers of their generation, can be formed using constraints which can limit the residual vibration of the system to zero at the modeled natural frequency and damping ratio, provided there is no error in the modeled frequency. The zero-vibration solution leads us to set $V\left(\omega_{n}, \zeta\right)=0$ and solve for the variables of the shaper. Thus, these shapers are typically known as Zero Vibration (ZV) shapers (Peláez et al 2005), which in compact form can be written as

$$
\left[\begin{array}{c}
A_{i} \\
t_{i}
\end{array}\right]_{2}=\left[\begin{array}{cc}
\frac{1}{1+M} & \frac{M}{1+M} \\
0 & \frac{\pi}{\omega_{d}}
\end{array}\right] .
$$

where $M=e^{-\frac{\zeta \pi}{\sqrt{1-\zeta^{2}}}}, \omega_{n}$ and $\zeta$ are the fundamental frequency and damping ratio of the regulator $\left(\mathbf{A}-\mathbf{B K}_{o p t}\right)$ respectively and $T_{d}$ is the damped period of vibration. Higher vibration modes can also be suppressed by convolving each of the impulse sequences obtained independently for a specific mode. The ZV shaper is highly susceptible to modeling errors and parametric uncertainties. That is why it is being called as a non-robust shaper.

5.5b Zero vibration and derivative (ZVD) shaper: The success of input shaping technique depends purely on the accuracy of the mathematical model of the physical system. Due to parametric uncertainties and modeling inaccuracies, deriving an accurate mathematical model is quite challenging. In such a scenario, input shaping will not produce a zero vibration response. The input shaper can be made insensitive to errors in natural frequencies of the system by setting $\frac{\partial V\left(\omega_{n}, \zeta\right)}{\partial \omega_{n}}=0$. Solving for the shaper parameters after the addition of this constraint results in a three-impulse sequence, which is also known as Zero Vibration and Derivative (ZVD) shaper. The ZVD shaper (Singer \& Seering 1990) is given as

$$
\left[\begin{array}{c}
A_{i} \\
t_{i}
\end{array}\right]_{3}=\left[\begin{array}{ccc}
\frac{1}{1+2 M+M^{2}} & \frac{2 M}{1+2 M+M^{2}} & \frac{M^{2}}{1+2 M+M^{2}} \\
0 & \frac{\pi}{\omega_{d}} & \frac{2 \pi}{\omega_{d}}
\end{array}\right] .
$$

This shaper is typically used where a fair amount of uncertainty cannot be neglected in the system parameters. It can be seen that the increase in robustness of ZVD shaper is earned at the cost of a marginal time penalty, which increases the time-lag of the system. The length (duration) of the ZVD shaper is exactly twice that of the ZV shaper.

5.5c Zero vibration and double derivative (ZVDD) shaper: The robustness of the input shaper can be further increased by taking one step ahead i.e. setting $\frac{\partial^{2} V\left(\omega_{n}, \zeta\right)}{\partial \omega_{n}^{2}}=0$, which results in a 
four-impulse sequence (Vaughan et al 2008). This sequence is also known as Zero Vibration and Double Derivative (ZVDD) shaper, which is given as

$$
\left[\begin{array}{c}
A_{i} \\
t_{i}
\end{array}\right]_{4}=\left[\begin{array}{cccc}
\frac{1}{1+3 M+3 M^{2}+M^{3}} & \frac{3 M}{1+3 M+3 M^{2}+M^{3}} & \frac{3 M^{2}}{1+3 M+3 M^{2}+M^{3}} & \frac{M^{3}}{1+3 M+3 M^{2}+M^{3}} \\
0 & \frac{\pi}{\omega_{d}} & \frac{2 \pi}{\omega_{d}} & \frac{3 \pi}{\omega_{d}}
\end{array}\right]
$$

\section{Results and discussion}

In the current section, a single-link flexible manipulator is considered, as shown in figure 1. The control objective is to track the large hub angle trajectory, while maintaining low vibration levels. The manipulator is modeled with finite-element approach using five elements. In this case study, the manipulator is controlled under open-loop as well as closed-loop control scheme. In the open-loop control, the aim is to test the effectiveness of command shaping in vibration reduction, whereas closed-loop control is aimed at tracking the large hub-angle trajectory while maintaining low vibration levels. Before going to the control phase, the dynamic model of the single-link flexible manipulator is validated.

\subsection{Open-loop control scheme}

After validating the model in the previous section, the current section presents the open-loop control of the manipulator. To obtain more realistic model, five elements are considered in the FEM model for simulation runs. The aim of the open-loop control (feedforward) scheme is to show the effectiveness of command shaping in reducing the vibration levels of the flexible system. Thus, using five elements, the total degree 5 s of freedom of the system are $2 n+3=$ $2 \times 5+3=13$ as given in (14), i.e. $\tilde{\mathbf{q}}=\left[\boldsymbol{\theta}, \overline{\boldsymbol{\psi}}^{\boldsymbol{T}}\right]^{T}$ and $\bar{\psi}=\left[u_{1}, u_{2}, u_{3}, \ldots, u_{2 n+1}, u_{2 n+2}\right]^{T}$. The manipulator is capable of bending in horizontal plane only. After applying boundary conditions, i.e. $u_{1}=u_{2}=0$ by appropriately deleting rows and columns, the total degrees of freedom of the system becomes $2 n+1$ i.e. $\mathbf{q}=\left[\theta, \psi^{T}\right]^{T}$ and $\psi=\left[u_{3}, u_{4}, \ldots, u_{2 n+1}, u_{2 n+2}\right]^{T}$, as given in (20).

Using the Lagrange approach, the complete dynamic model of the single-link flexible manipulator is calculated, as explained in Section 2, and can be represented in the standard form $M(q) \ddot{q}+n(q, \dot{q})=\tau$, as given in (40). After linearizing the system, the dynamic model can be represented in state-space form as $\dot{\mathbf{x}}=A x+B u$, where the state-vector of dimension $22 \times 1$

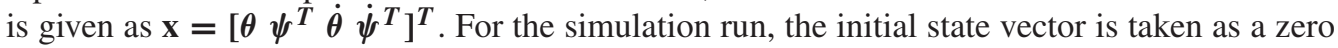
vector and external force vector is taken as $\tau=[\tau, 0,0, \ldots, 0]^{T}$ of appropriate dimensions. The first element of the external-force vector represents the torque applied at the hub of the singlelink flexible manipulator. To induce more vibration, the torque applied at the hub is chosen as a single-switch bang-bang type with amplitude of $0.3 \mathrm{Nm}$, as shown in figure 4.

The command shapers are designed on the basis of model parameters, i.e. resonant frequencies and damping ratios of the system. Using the Matlab damp function, resonant frequencies of the first three dominated modes are found as $13.31,35.11$ and $64.83 \mathrm{~Hz}$. The damping ratios are assumed as $0.026,0.038$ and 0.04 respectively, as reported in Mohamed \& Tokhi (2002). It is also shown in figure 4 , how the input torque gets modified with the application of one-mode, twomode and three-mode shapers. With the above mentioned system parameters $\left(\omega_{n}, \zeta\right)$, the ZVDD shaper parameters are calculated using (75) and are given in table 3 . The above table summarizes the shaper parameters calculated for two-impulse (ZV shaper) and four-impulse (ZVDD shaper) 


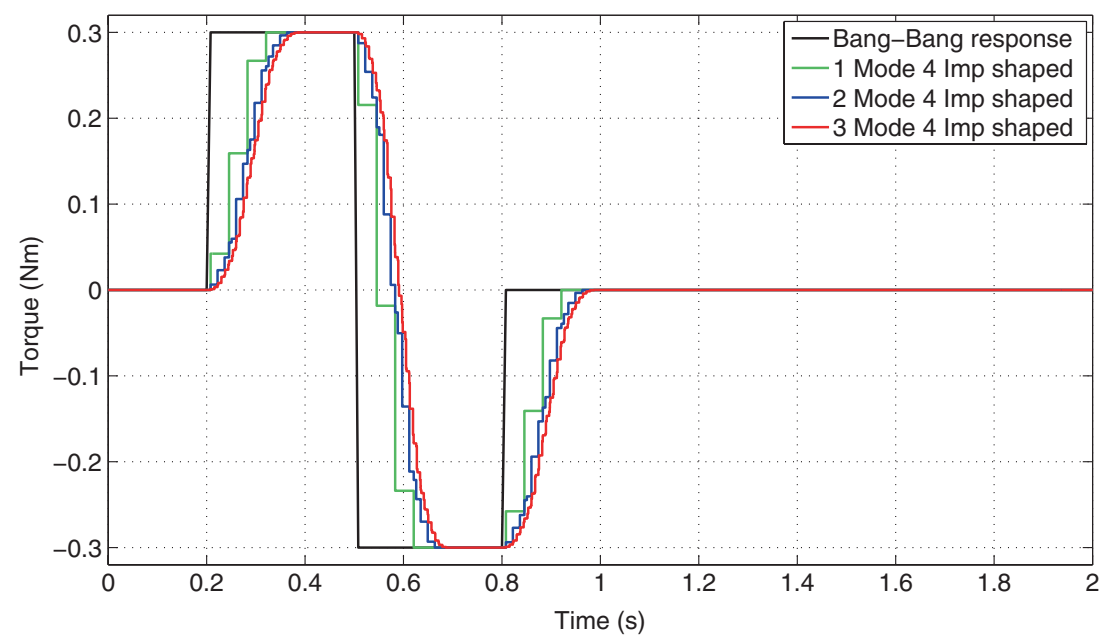

Figure 4. Bang-bang input vs 1/2/3-mode, four-impulse shaped input to the single-link flexible manipulator.

sequence, which are found to be in close agreement with the corresponding values reported in Mohamed \& Tokhi (2002). After developing the dynamic model, state-space representation and shaper parameters, it is the time to start with the first simulation run.

The first simulation run presents the single switch, bang-bang, unshaped response of the single-link flexible manipulator, as shown in figure 5. Duty-cycle of the input torque is $0.6 \mathrm{~s}$, which end at time $t=0.8 \mathrm{~s}$. However, to critically examine the vibration levels, the simulation is run for 2 seconds. With the application of input torque, the endpoint displacement is found as $0.6 \mathrm{~m}$ as shown in figure 5(a). The endpoint residual is shown in figure 5(b), which is oscillating about the mean position. The steady-state hub angle value of the manipulator is found as $38^{\circ}$, as shown in figure 5(c) and hub velocity is shown in figure 5(d). As no damping is considered in the model, all the plots show significant amount of oscillations.

As shown in the above figures, the endpoint residual and hub velocity are found to oscillate between -0.04 to 0.05 and -350 to $350 \mathrm{~m} / \mathrm{s}$, respectively. Figure 6 shows the comparison of bang-bang vs first-mode shaped response of the manipulator. As expected, vibration level in all the plots is reduced significantly. The maximum endpoint displacement, which is reported as $0.6017 \mathrm{~m}$ for the bang-bang response, is reduced to $0.5986 \mathrm{~m}$. Similar trend is observed in

Table 3. Shaper parameters of a single-link flexible manipulator calculated for $\omega_{n}=\{13.31,35.11$, $64.83\}^{T}(\mathrm{~Hz})$ and $\zeta=\{0.026,0.038,0.040\}^{T}$.

\begin{tabular}{|c|c|c|c|c|c|c|c|}
\hline \multirow[t]{2}{*}{ Shaper } & \multirow{2}{*}{$\begin{array}{c}\text { Impulse } \\
(i)\end{array}$} & \multicolumn{2}{|c|}{ First mode } & \multicolumn{2}{|c|}{ Second mode } & \multicolumn{2}{|c|}{ Third mode } \\
\hline & & $\overline{\text { Magnitude }}$ & Time & Mag. & Time & Mag. & Time \\
\hline \multirow[t]{3}{*}{$\overline{\mathrm{ZV}}$} & 1 & 0.5204 & 0.0000 & 0.5298 & 0.0000 & 0.5314 & 0.0000 \\
\hline & 2 & 0.4796 & 0.0376 & 0.4702 & 0.0143 & 0.4686 & 0.0077 \\
\hline & 1 & 0.1409 & 0.0000 & 0.1487 & 0.0000 & 0.1500 & 0.0000 \\
\hline \multirow[t]{2}{*}{ ZVDD } & 2 & 0.3897 & 0.0376 & 0.3960 & 0.0143 & 0.3970 & 0.0077 \\
\hline & 3 & 0.3591 & 0.0752 & 0.3514 & 0.0285 & 0.3501 & 0.0154 \\
\hline 4 & 0.1103 & 0.1127 & 0.1039 & 0.0428 & 0.1029 & 0.0232 & \\
\hline
\end{tabular}




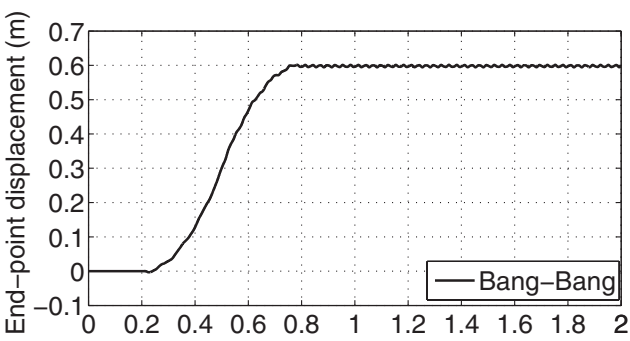

(a)

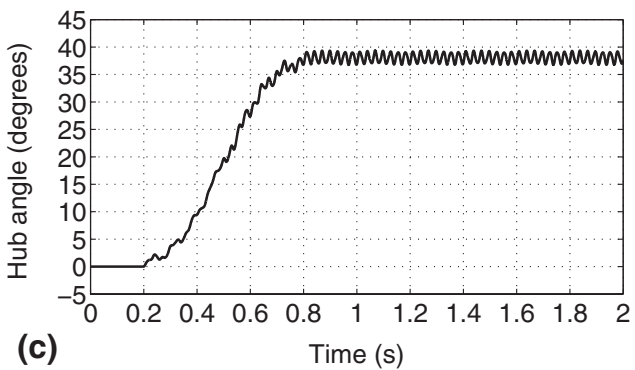

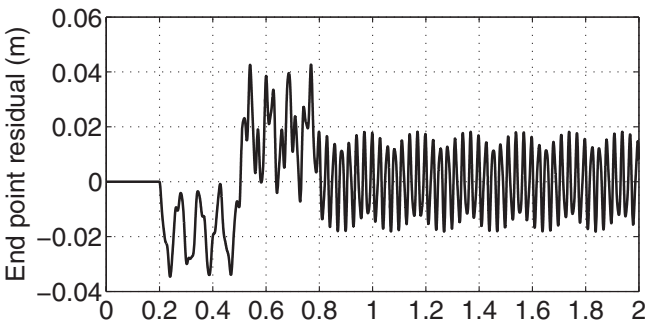

(b)

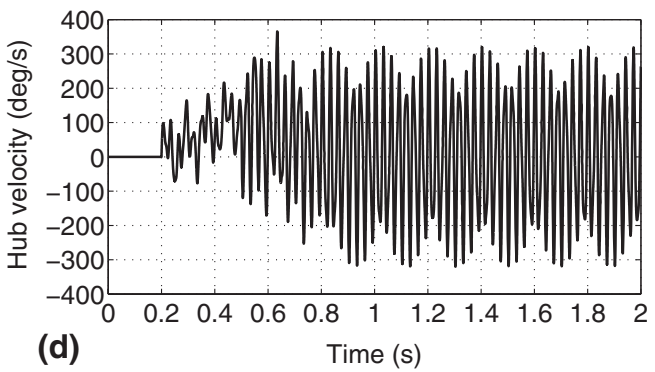

Figure 5. Bang-bang response of the single-link flexible manipulator using five elements.

the remaining three plots, where maximum end-point residual, hub angle and hub velocity are reported as $0.0426 \mathrm{~m}, 39.36^{\circ}$ and $365.50 \mathrm{~m} / \mathrm{s}$ for the bang-bang case are reduced to $0.0196 \mathrm{~m}$, $38.24^{\circ}$ and $140.82 \mathrm{~m} / \mathrm{s}$ in the first-mode shaped response, respectively. Precisely, with the firstmode shaping, there is a $0.51 \%$ reduction at the peak end-point displacement level, $53.97 \%$ at

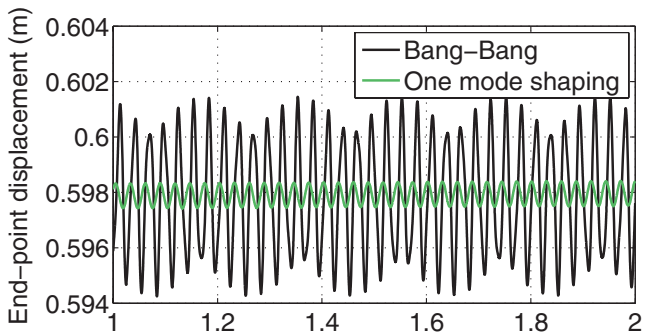

(a)

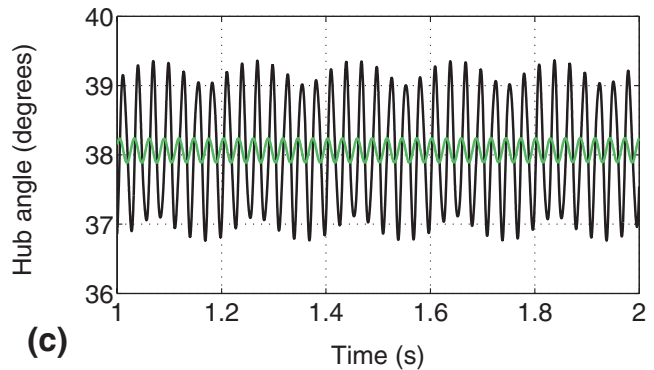

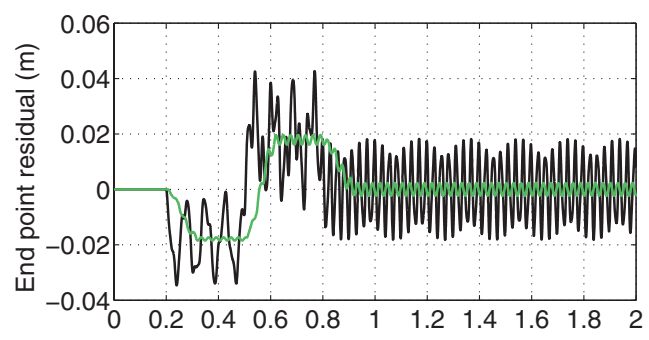

(b)

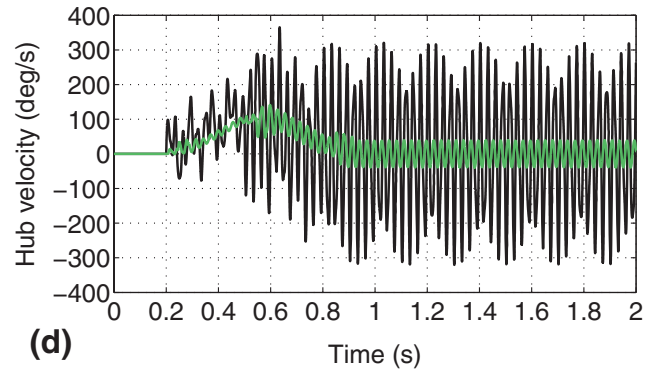

Figure 6. Bang-bang vs single-mode shaped response of the single-link flexible manipulator. 


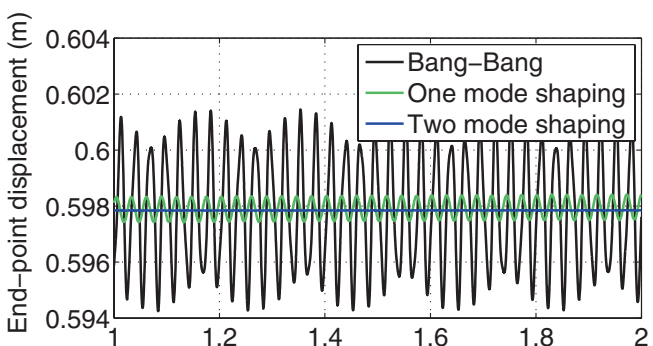

(a)

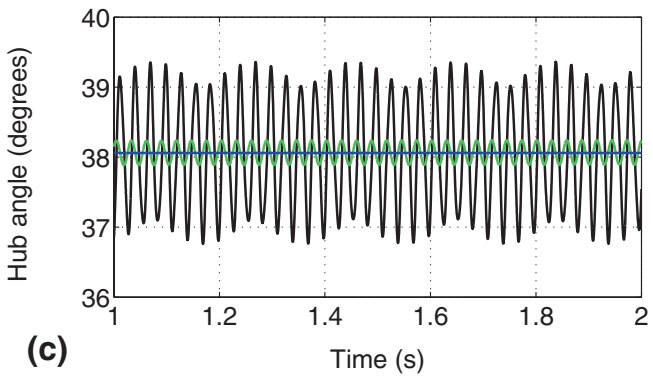

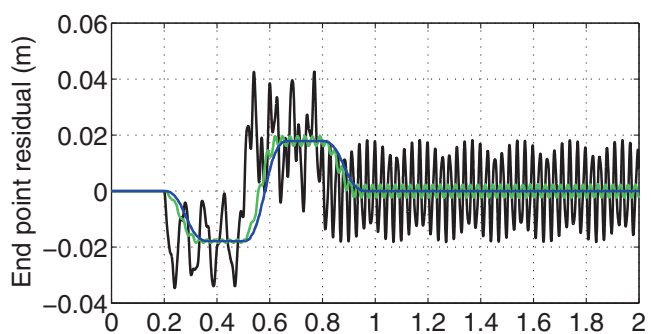

(b)

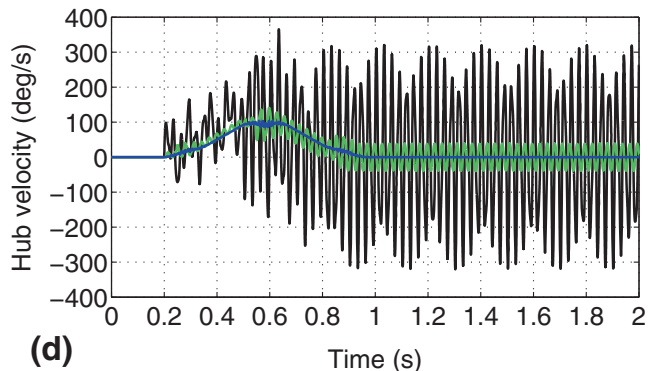

Figure 7. Bang-bang vs 1/2-mode shaped response of the single-link flexible manipulator.

the peak endpoint residual level, $2.84 \%$ at the peak hub-angle level and $61.47 \%$ at the peak hub-angle velocity level.

The small amount of vibrations left in the first-mode shaped response is further reduced with two-mode and three-mode shaping processes, as shown in figures 7 and 8 respectively.

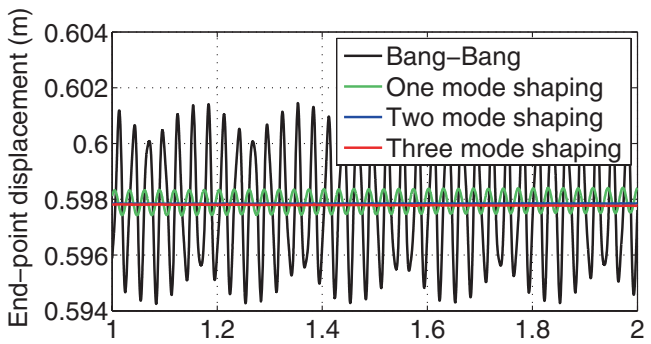

(a)

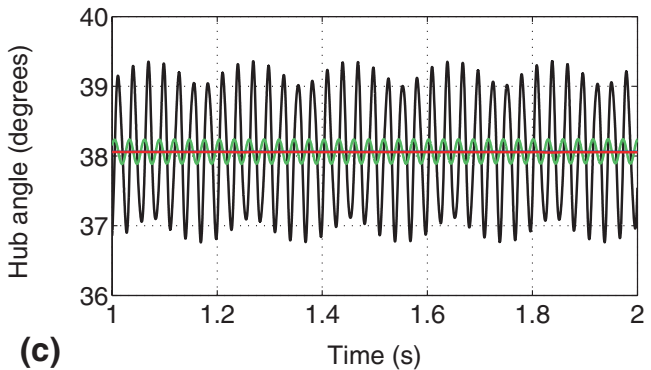

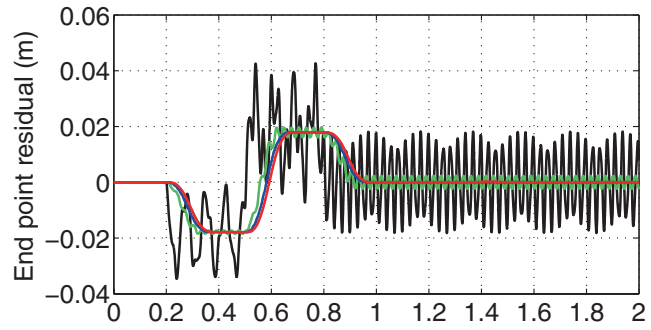

(b)

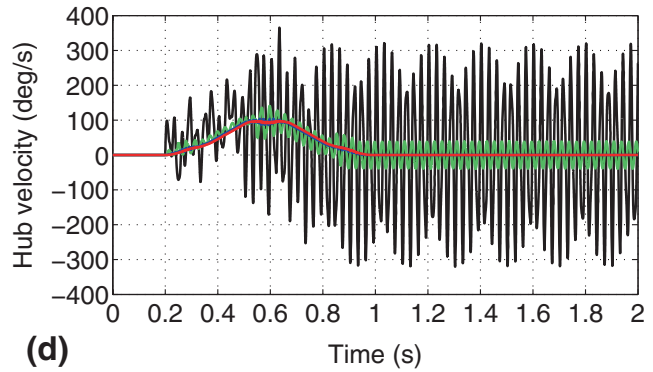

Figure 8. Bang-bang vs 1/2/3-mode shaped response of the single-link flexible manipulator. 


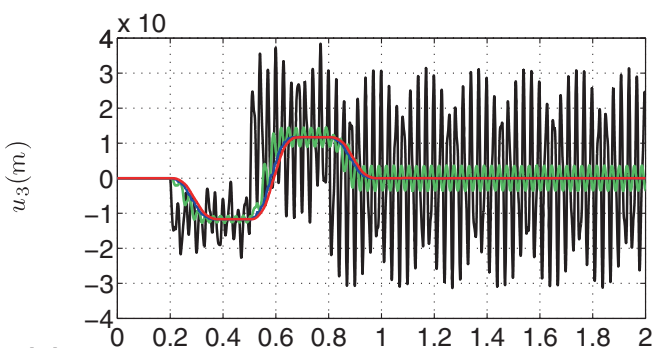

(a)

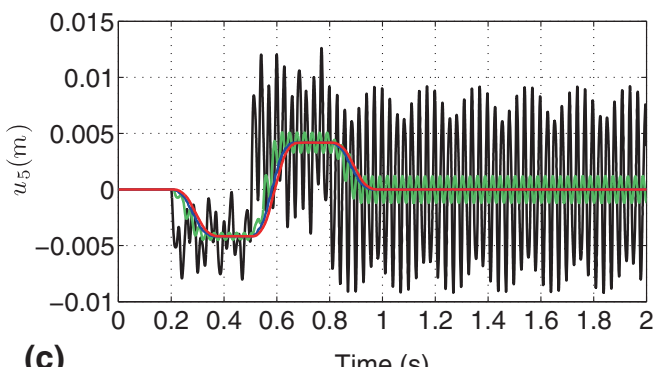

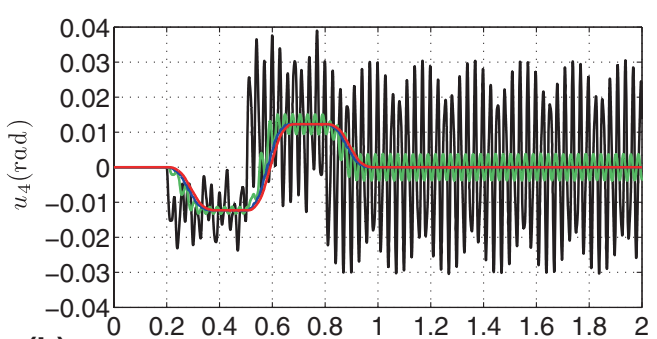

(b)

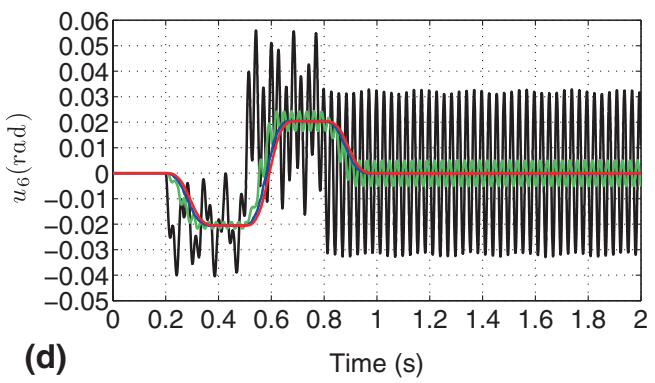

Figure 9. Bang-bang vs 1/2/3-mode shaped flexural response of the single-link flexible manipulator.

Comparison of bang-bang vs 1/2/3-mode shaped response of first few flexural modes are shown in figure 9. All the results are summarized in table 4. It can be clearly seen from the table that with two-mode shaping, vibration levels are further reduced as compared to the single-mode shaping. There is a $0.626 \%$ reduction at the peak end-point displacement level, $58.06 \%$ at the peak endpoint residual level, 3.30\% at the peak hub-angle level and $71.52 \%$ at the peak hubangle velocity level. Further, it can be observed that there is a marginal gain at all the levels with the three-mode shaping process. This is due to the high frequency of the third mode $(64.83 \mathrm{~Hz})$ as compared to the frequency of the second flexural mode $(35.11 \mathrm{~Hz})$. Thus, it can be concluded that the third mode is not dominant and only first two modes are sufficient enough to reduce the vibration levels significantly.

Table 4. Simulation Results: comparison of reduction in vibration levels of bang-bang vs 1/2/3-mode shaped response.

\begin{tabular}{|c|c|c|c|c|c|c|c|}
\hline \multirow[t]{2}{*}{ Variable } & \multirow{2}{*}{$\begin{array}{c}\text { Bang-Bang } \\
\text { response }\end{array}$} & \multicolumn{2}{|c|}{ 1-Mode shaper } & \multicolumn{2}{|c|}{ 2-Mode shaper } & \multicolumn{2}{|c|}{ 3-Mode shaper } \\
\hline & & Value & $\%$ Red. & Value & $\%$ Red. & Value & $\%$ Red \\
\hline$\left|y_{\text {tip }}(t)_{\max }\right|$ & 0.6071 & 0.5986 & 0.513 & 0.5980 & 0.626 & 0.5979 & 0.633 \\
\hline$\left|\theta(t)_{\max }\right|$ & 39.360 & 38.243 & 2.838 & 38.062 & 3.298 & 38.059 & 3.306 \\
\hline$\left|\dot{\theta}(t)_{\max }\right|$ & 365.50 & 140.82 & 61.473 & 104.08 & 71.523 & 98.025 & 73.181 \\
\hline$\left|u_{3}(t)_{\max }\right|$ & $3.839 \mathrm{e}-3$ & $1.448 \mathrm{e}-3$ & 62.27 & $1.175 \mathrm{e}-3$ & 69.39 & $1.172 \mathrm{e}-3$ & 69.48 \\
\hline$\left|u_{4}(t)_{\max }\right|$ & $3.897 \mathrm{e}-2$ & $1.512 \mathrm{e}-2$ & 61.19 & $1.232 \mathrm{e}-2$ & 68.38 & $1.230 \mathrm{e}-2$ & 68.43 \\
\hline$\left|u_{5}(t)_{\max }\right|$ & $1.261 \mathrm{e}-2$ & $5.077 \mathrm{e}-3$ & 59.73 & $4.183 e-3$ & 66.82 & $4.179 \mathrm{e}-3$ & 66.85 \\
\hline$\left|u_{6}(t)_{\max }\right|$ & $5.600 \mathrm{e}-2$ & $2.426 \mathrm{e}-2$ & 56.67 & $2.046 \mathrm{e}-2$ & 63.46 & $2.045 \mathrm{e}-3$ & 63.48 \\
\hline
\end{tabular}




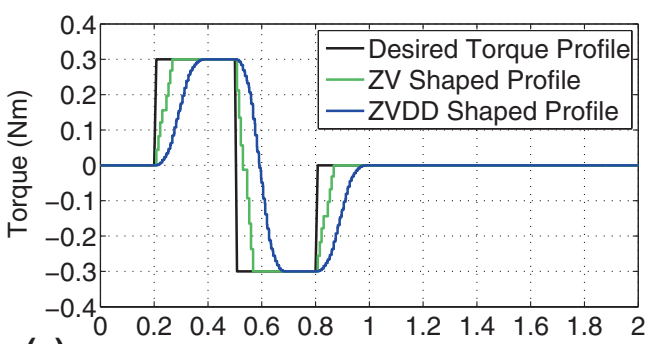

(a)

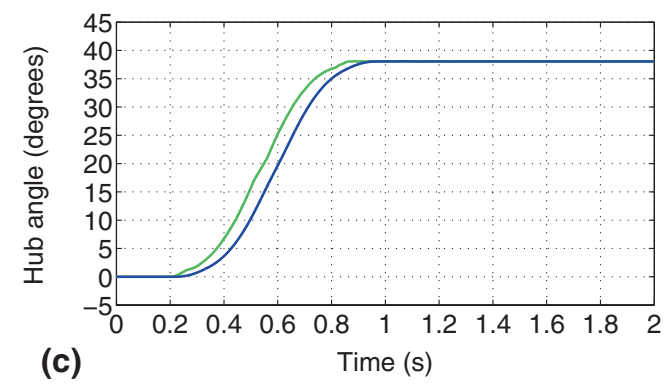

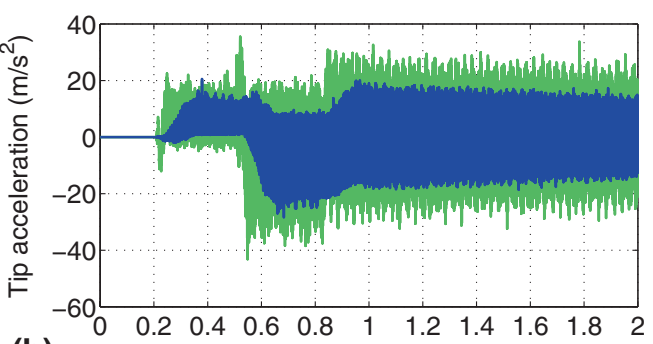

(b)

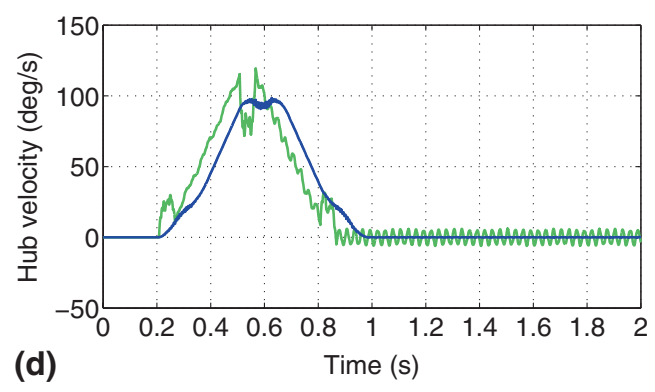

Figure 10. Comparison of three-mode, two-impulse (ZV) shaped vs four-impulse (ZVDD) shaped response.

\subsection{Effect of payload on the performance of the shaper}

In this subsection, the robustness of two different types of shapers is evaluated. While performing in real-world applications, the manipulator may come across different situations in which the mass acting on the tip of the manipulator changes, which can cause the parameters of the manipulator to change. As the command shaping is very sensitive to parametric variations, it becomes very important to analyze the situation so that correct selection of the shaper for a particular application can be made. First of all, the response of two-impulse (ZV) shaper and four-impulse (ZVDD) shaper is calculated based on the shaper parameters given in table 3 .

Figure 10(a) shows the bang-bang input torque profile to the manipulator. The figure also shows how the input profile to the system gets modified due to the application of ZV and ZVDD shaper. It can be clearly seen that the input to the system as well as the hub-angle response, as shown in figure $10(\mathrm{c})$, gets delayed by $0.0596 \mathrm{~s}(0.1787 \mathrm{~s})$ which is equal to length of the $\mathrm{ZV}$ (ZVDD) shaper. In other words, it can be said that the ZVDD shaped profile is further delayed by $0.1787-0.0596=0.1191 \mathrm{~s}$ from the $\mathrm{ZV}$ shaped profile. The marginal time penalty paid by the ZVDD shaper is justified in figure 10(b) and (d), which show the tip-acceleration response and

Table 5. Variation in first-three resonant frequencies $(\mathrm{Hz})$ reported as percentage error, calculated using 5 elements, due to different payloads acting at the tip of the single-link flexible manipulator.

\begin{tabular}{|c|c|c|c|c|c|c|c|}
\hline \multirow{2}{*}{$\begin{array}{l}\text { Mode } \\
(i)\end{array}$} & \multirow{2}{*}{$\begin{array}{l}\text { No payload } \\
\text { Freq. }\end{array}$} & \multicolumn{2}{|c|}{$1 \mathrm{gm}$ payload } & \multicolumn{2}{|c|}{$10 \mathrm{gm}$ payload } & \multicolumn{2}{|c|}{$25 \mathrm{gm}$ payload } \\
\hline & & Freq. & Error & Freq. & Error & Freq. & Error \\
\hline 1 & 13.31 & 13.03 & 2.10 & 10.98 & 17.51 & 8.81 & 33.81 \\
\hline 2 & 35.15 & 35.13 & 0.05 & 35.13 & 0.05 & 33.27 & 5.35 \\
\hline 3 & 65.04 & 60.62 & 6.80 & 40.29 & 38.05 & 35.13 & 45.98 \\
\hline
\end{tabular}


hub-velocity response, respectively. It can be clearly seen that vibration levels are significantly reduced in the case of ZVDD response. To be precise, the maximum tip acceleration is found as $35.5 \mathrm{~m} / \mathrm{s}^{2}$ in the $\mathrm{ZV}$ shaped response, which is reduced to $20.5 \mathrm{~m} / \mathrm{s}^{2}$, resulting in $42.25 \%$ reduction in the maximum tip acceleration value. Similarly, hub-velocity is found as $119.5 \mathrm{deg} / \mathrm{s}$ in the ZV shaped response, which is reduced to $98.4 \mathrm{deg} / \mathrm{s}$ in the case of ZVDD response, with an overall reduction of $17.66 \%$.

The next simulation run shows the comparison of $\mathrm{ZV}$ shaped responses under the application of different payloads. As mentioned earlier, the payload (tip mass) disturbs the system parameters significantly. This can be verified with the data shown in table 5, which reports a 30-40\% change in the resonant frequencies of the single-link flexible manipulator under the presence of different payloads. The poor robustness of the ZV shaper to this parametric changes is evident in figure 11, which shows that as the payload on the tip of manipulator is increasing, the performance of the $\mathrm{ZV}$ shaper is decreasing. More vibrations can be found in all the three responses, i.e. hub-angle, hub-velocity and the tip acceleration.

As reported in table 5, frequency changes are marginal under the $1 \mathrm{gm}$ payload case, that is why the response in this category is almost similar to the no payload condition, whereas in the case of $10 \mathrm{gm}$ and $25 \mathrm{gm}$ payload conditions, the oscillations are increased significantly in the hub-velocity response, as shown in figure 11(b). In the case of no payload condition, i.e. with exact resonant frequencies, the maximum hub-velocity is found as $119.46 \mathrm{deg} / \mathrm{s}$, which increases to $124.54 \mathrm{deg} / \mathrm{s}$ in the case of $10 \mathrm{gm}$ payload, and further to $145.30 \mathrm{deg} / \mathrm{s}$ in the case of $25 \mathrm{gm}$ payload. The vibrations at velocity level are increased by $4.25 \%$ with 10 gm tip mass and by $21.63 \%$ with 25 gm tip mass. Similar trends can be observed in the tip-acceleration response, as shown in figure 11(c), which reports that the vibrations at acceleration level are increased by $25.93 \%$ with 10 gm tip load and by $33.10 \%$ with 25 gm tip load, respectively.

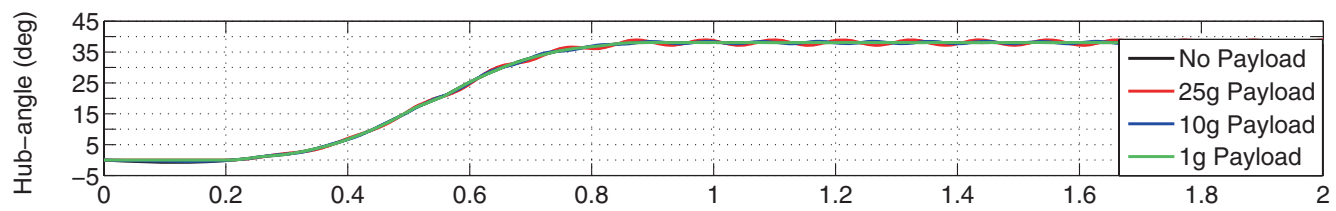

(a)

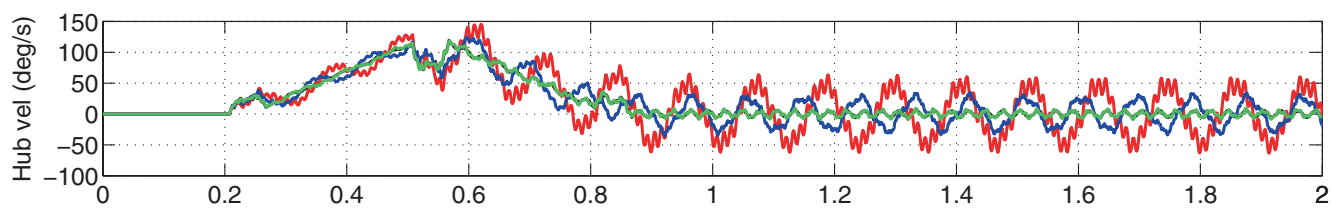

(b)

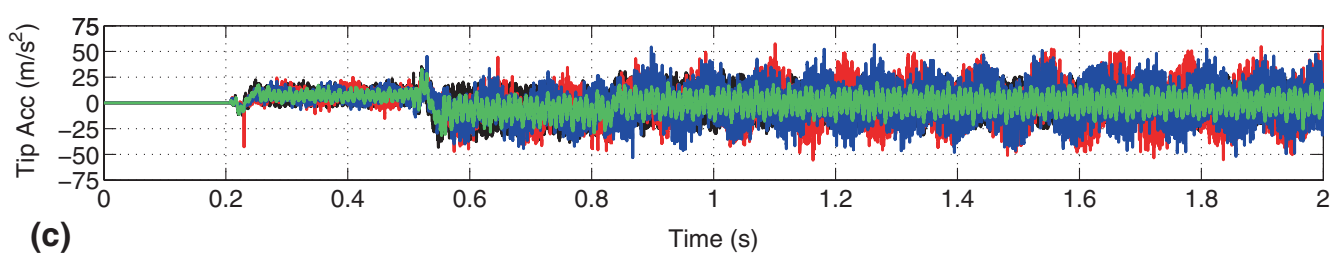

Figure 11. Three-mode response of the two-impulse $(\mathrm{ZV})$ shaper to variations in the tip mass of the single-link flexible manipulator. 
The last simulation run in this category shows how ZVDD shaper copes up with variations in system parameters due to the application of different payloads. It can be clearly observed in figure 12 that ZVDD shaper gives a much smoother response in spite of parametric variations as compared to the ZV shaper. It can be clearly seen that the vibration levels increase marginally at the hub-velocity level, whereas the levels are of the same magnitude at the tip-acceleration level. The maximum hub-velocity has increased slightly from $98.3 \mathrm{deg} / \mathrm{s}$ with no payload condition to $103.7 \mathrm{deg} / \mathrm{s}$ with $25 \mathrm{gm}$ payload, whereas the maximum tip-acceleration has increased marginally from $28.34 \mathrm{~m} / \mathrm{s}^{2}$ with no payload condition to $28.5 \mathrm{~m} / \mathrm{s}^{2}$ with $25 \mathrm{gm}$ payload. In terms of relative performance of both the shapers, it can be observed that with 25 gm payload condition, the vibration levels are increased by $5.49 \%$ with ZVDD shaper as compared to $21.63 \%$ with ZV shaper at the hub-velocity level, whereas the vibrations are increased by $0.56 \%$ with ZVDD shaper as compared to $33.10 \%$ with the ZV shaper at the tip-acceleration level. Thus, it can be concluded that the ZVDD shaper is more robust to parametric variations as compared to ZV shaper.

\subsection{Closed-loop control scheme}

In this section, comparison of two closed-loop control schemes is presented. The closed-loop control scheme is developed for rigid-body motion control as well as vibration suppression of the single-link flexible manipulator. For vibration suppression, ZVDD shaper is chosen, as concluded in the previous section. The control objective is to track a large hub-angle trajectory with minimum vibration levels. The large hub-angle trajectory means that hub-angle movement is not in the linear range, and maximum hub-angle change is of the order of $\pm 80^{\circ}$, as shown in figure 13. As in the case of open-loop control, the flexible manipulator is considered as a hingedfree beam and is assumed to be made of five elements, leading to 11 degrees of freedom of the system after applying the appropriate boundary conditions.

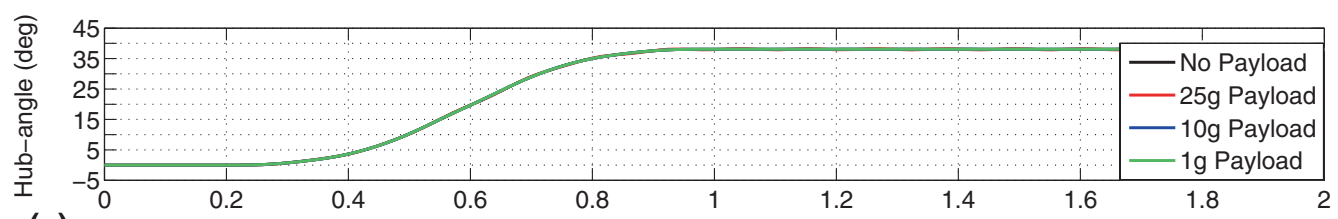

(a)

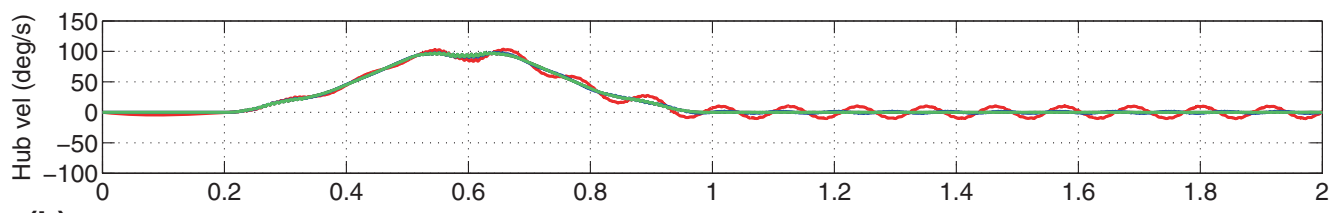

(b)

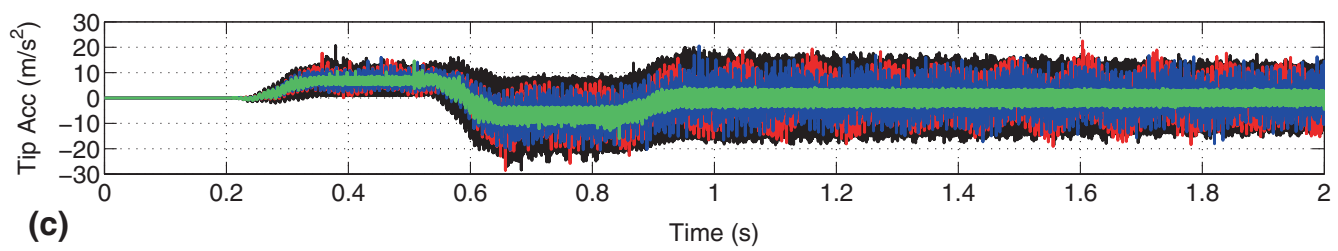

Figure 12. Three-mode response of the four-impulse (ZVDD) shaper to variations in the tip mass of the single-link flexible manipulator. 

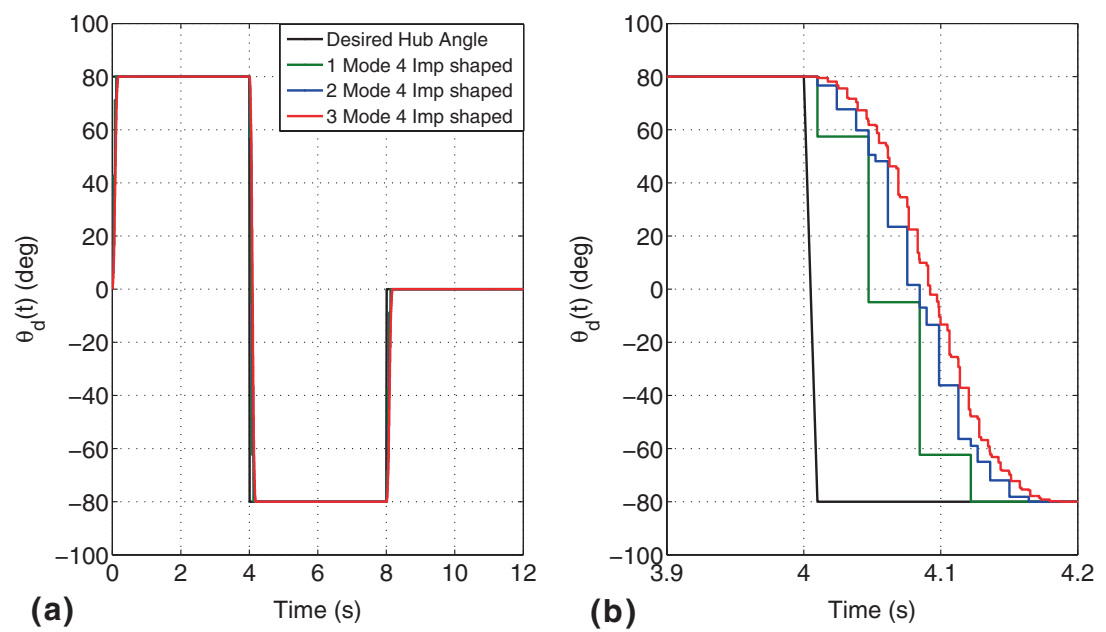

Figure 13. Bang-bang vs $1 / 2 / 3$-mode, four-impulse shaped desired hub-angle trajectory to be tracked by the single-link flexible manipulator.

All the parameters of the flexible manipulator are kept the same, as given in table 1. Only area moment of inertia is taken as $I=5.1924 \times 10^{-11} \mathrm{~m}^{4}$, as reported in Zain et al (2006a) and Zain et al (2006b). Due to this change, the first three resonant frequencies are increased slightly to $13.39,35.11$ and $65.20 \mathrm{~Hz}$ as compared to those given in table 2, whereas the corresponding values are reported as $13.47,35.43$ and $65.37 \mathrm{~Hz}$ respectively by Zain et al (2006a). The resonant frequencies are found to be in close agreement, which validates the accuracy of the dynamic model. The damping ratios are slightly altered to $\zeta=\{0.026,0.038,0.050\}^{T}$. Due to small variations in the values of $\omega_{n}$ and $\zeta$, shaper parameters are changed marginally to those calculated in table 3 and are not reported again. However, the effect of new shaper parameters in modifying the input to the system is shown in figure 13, which shows the bang-bang vs shaped hub-angle $\left(\theta_{d}(t)\right)$ reference trajectory to be tracked by the single-link flexible manipulator.

As mentioned earlier, the closed-loop scheme presents the comparison of two different control schemes - collocated PD control and reduced-order observer based shaped LQR control. The collocated PD control is used by Zain et al (2006b) and is based on the PD feedback of collocated sensor signals. The PD controller is implemented by linearizing the dynamic model around the nominal trajectory to obtain the state-space representation. As mentioned earlier in the openloop control case, only first two modes are found to be dominant. The control input given by Zain et al is given as

$$
u(s)=A_{c}\left[K_{p}\left\{\theta_{d}(s)-\theta(s)\right\}-K_{d} s \theta(s)\right],
$$

where

$$
\begin{aligned}
s & =\text { Laplace variable, } \\
\theta_{d} & =\text { reference hub-angle, } \\
\theta & =\text { actual hub-angle, } \\
K_{p} & =\text { proportional gain } \\
K_{d} & =\text { derivative gain, } \\
A_{c} & =\text { motor amplifier gain. }
\end{aligned}
$$


The PD controller is designed on the basis of root-locus analysis and the three gains are reported as $K_{p}=0.64, K_{d}=0.32$ and $A_{c}=0.01$. However, in the case of LQR-ROO-MS scheme, the feedback design is based on optimal control theory. With 11 DOFs, the dimension of

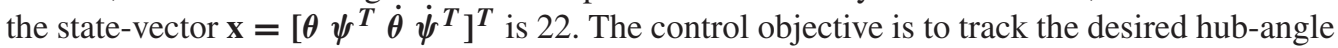
trajectory. Therefore the desired state-vector is $\mathbf{x}=\left[\theta(t) \mathbf{0}_{1 \times 21}\right]^{T}$.

Eigenvalues of the open-loop plant $\left(\lambda_{O L}\right)$, reported in table 6 , show the presence of a double pole at the origin, which clearly indicates that the plant is unstable and cannot be controlled with feedforward control only. Thus, the feedback term $\mathbf{u}_{f b}$ is required to stabilize the plant and to maintain the required positional accuracy. The feedback term is calculated using optimal control and the LQR parameters are given in table 6. Using these values, gains of optimal regulator $\left(\mathbf{K}_{\text {opt }}\right)$ are calculated. Further, eigen values of the closed-loop system $\left(\mathbf{A}-\mathbf{B} \mathbf{K}_{o p t}\right)$, i.e. $\left(\lambda_{C L}\right)$ are calculated and found to have negative real parts, which justifies the selection of optimal gains and ensures asymptotic stability of the closed-loop system.

In designing the reduced-order observer, hub-angle is taken as the only output variable. Thus, the state-vector in partitioned form can be represented as

$$
\mathbf{x}(t)=\left\{\mathbf{x}(t)^{T}, \mathbf{x}_{2}(t)^{T}\right\}^{T}, \text { where } \mathbf{x}(t)=\{\theta(t)\} \text { and } \mathbf{x}_{2}(t)=\left\{\psi^{T} \dot{\theta} \dot{\psi}^{T}\right\}^{T} .
$$

All the observer poles are placed deeply in the left-half plane by choosing $\mathbf{v}=5 \times \lambda_{C L}$. Finally, the shaper parameters of the four-impulse ZVDD shaper are calculated with the given values of $\omega_{n}$ and $\zeta$. Figure 14 shows the comparison of hub-angle tracking response obtained with collocated PD control vs 1/2/3-mode shaped LQR control. Hub-angle tracking seems to be

Table 6. Controller parameters of the single-link flexible manipulator.

\begin{tabular}{lcccccc}
\hline Var. & $\mathbf{x}(0)$ & $\mathbf{x}_{2}(0)$ & $\mathrm{Q}$ & $\lambda_{O L}$ & $\lambda_{C L}$ & $\mathbf{K}_{\text {opt }}$ \\
\hline$\theta$ & 0 & - & 5 & 0 & $-2.33 \mathrm{e} 0+1.57 \mathrm{e} 0 j$ & $3.16 \mathrm{e}-1$ \\
$u_{3}$ & 0 & 0.01 & 0 & 0 & $-2.33 \mathrm{e} 0-1.57 \mathrm{e} 0 j$ & $1.28 \mathrm{e}-2$ \\
$u_{4}$ & 0 & 0.01 & 0 & $+8.41 \mathrm{e} 1 j$ & $-1.33 \mathrm{e} 1+8.53 \mathrm{e} 1 j$ & $1.53 \mathrm{e}-4$ \\
$u_{5}$ & 0 & 0.01 & 0 & $-8.41 \mathrm{e} 1 j$ & $-1.33 \mathrm{e} 1-8.53 \mathrm{e} 1 j$ & $2.55 \mathrm{e}-2$ \\
$u_{6}$ & 0 & 0.01 & 0 & $+2.22 \mathrm{e} 2 j$ & $-4.23 \mathrm{e} 1+2.15 \mathrm{e} 2 j$ & $1.52 \mathrm{e}-4$ \\
$u_{7}$ & 0 & 0.01 & 0 & $-2.22 \mathrm{e} 2 j$ & $-4.23 \mathrm{e} 1-2.15 \mathrm{e} 2 j$ & $3.82 \mathrm{e}-2$ \\
$u_{8}$ & 0 & 0.01 & 0 & $+4.11 \mathrm{e} 2 j$ & $-1.93 \mathrm{e} 1+4.04 \mathrm{e} 2 j$ & $1.52 \mathrm{e}-4$ \\
$u_{9}$ & 0 & 0.01 & 0 & $-4.11 \mathrm{e} 2 j$ & $-1.93 \mathrm{e} 1-4.04 \mathrm{e} 2 j$ & $5.08 \mathrm{e}-2$ \\
$u_{10}$ & 0 & 0.01 & 0 & $+7.41 \mathrm{e} 2 j$ & $-5.37 \mathrm{e} 0+7.39 \mathrm{e} 2 j$ & $1.52 \mathrm{e}-4$ \\
$u_{11}$ & 0 & 0.01 & 0 & $-7.41 \mathrm{e} 2 j$ & $-5.37 \mathrm{e} 0-7.39 \mathrm{e} 2 j$ & $2.98 \mathrm{e}-2$ \\
$u_{12}$ & 0 & 0.01 & 0 & $+1.20 \mathrm{e} 3 j$ & $-1.76 \mathrm{e} 0+1.20 \mathrm{e} 3 j$ & $-8.76 \mathrm{e}-4$ \\
$\dot{\theta}$ & 0 & 0.01 & 0.5 & $-1.20 \mathrm{e} 3 j$ & $-1.76 \mathrm{e} 0-1.20 \mathrm{e} 3 j$ & $1.89 \mathrm{e}-1$ \\
$\dot{u}_{3}$ & 0 & 0.01 & 0 & $+1.98 \mathrm{e} 3 j$ & $-6.41 \mathrm{e}-1+1.99 \mathrm{e} 3 j$ & $1.17 \mathrm{e}-2$ \\
$\dot{u}_{4}$ & 0 & 0.01 & 0 & $-1.98 \mathrm{e} 3 j$ & $-6.41 \mathrm{e}-1-1.99 \mathrm{e} 3 j$ & $1.40 \mathrm{e}-4$ \\
$\dot{u}_{5}$ & 0 & 0.01 & 0 & $+2.90 \mathrm{e} 3 j$ & $-3.81 \mathrm{e}-1+2.90 \mathrm{e} 3 j$ & $2.33 \mathrm{e}-2$ \\
$\dot{u}_{6}$ & 0 & 0.01 & 0 & $-2.90 \mathrm{e} 3 j$ & $-3.81 \mathrm{e}-1-2.90 \mathrm{e} 3 j$ & $1.40 \mathrm{e}-4$ \\
$\dot{u}_{7}$ & 0 & 0.01 & 0 & $+4.21 \mathrm{e} 3 j$ & $-2.07 \mathrm{e}-1+4.21 \mathrm{e} 3 j$ & $3.50 \mathrm{e}-2$ \\
$\dot{u}_{8}$ & 0 & 0.01 & 0 & $-4.21 \mathrm{e} 3 j$ & $-2.07 \mathrm{e}-1-4.21 \mathrm{e} 3 j$ & $1.39 \mathrm{e}-4$ \\
$\dot{u}_{9}$ & 0 & 0.01 & 0 & $+5.97 \mathrm{e} 3 j$ & $-8.68 \mathrm{e}-2+5.97 \mathrm{e} 3 j$ & $4.65 \mathrm{e}-2$ \\
$\dot{u}_{10}$ & 0 & 0.01 & 0 & $-5.97 \mathrm{e} 3 j$ & $-8.68 \mathrm{e}-2-5.97 \mathrm{e} 3 j$ & $1.39 \mathrm{e}-4$ \\
$\dot{u}_{11}$ & 0 & 0.01 & 0 & $+8.77 \mathrm{e} 3 j$ & $-1.70 \mathrm{e}-3+8.78 \mathrm{e} 3 j$ & $2.73 \mathrm{e}-2$ \\
$\dot{u}_{12}$ & 0 & 0.01 & 0 & $-8.77 \mathrm{e} 3 j$ & $-1.70 \mathrm{e}-3-8.78 \mathrm{e} 3 j$ & $-8.03 \mathrm{e}-4$ \\
\hline
\end{tabular}




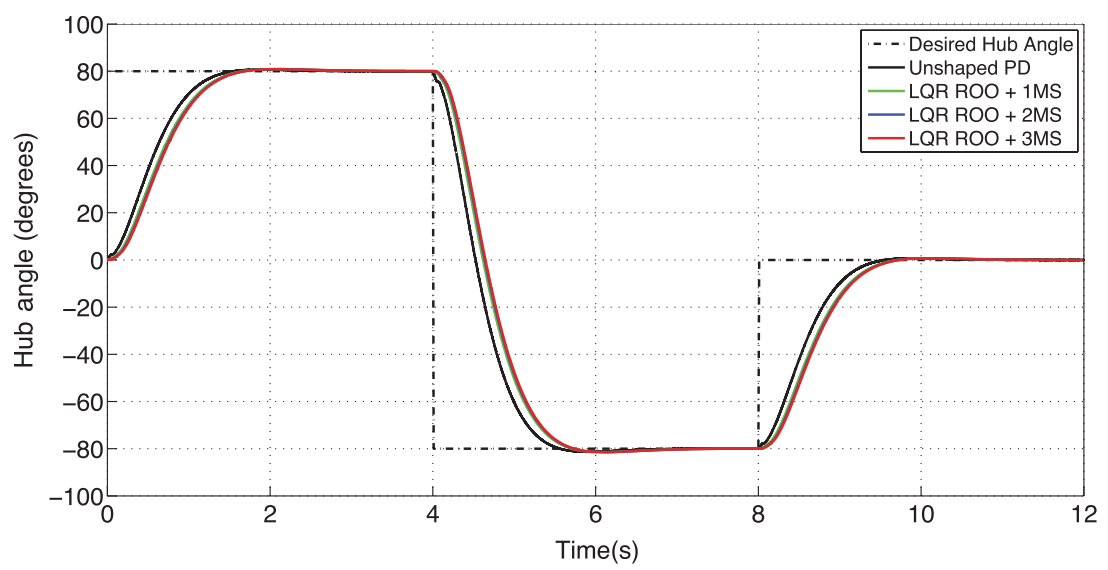

Figure 14. Comparison of unshaped vs shaped hub-angle (tracked) response.

reasonable in all the cases. As discussed in Section 5.5, command shaping comes with marginal time penalty, that is why the one-mode shaped response (shown in green color) is slightly delayed from the collocated PD response. The two-mode shaped response is further delayed. As the frequency of the third mode is higher $(65 \mathrm{~Hz})$, the delay in the three-mode shaped response is of the same order as that of the two-mode case.

The most significant parameters of the flexible manipulator, i.e. tip acceleration and control effort required, are shown in figure 15, which shows the comparison of these parameters obtained with collocated PD control and LQR-ROO-MS control. As the desired hub-angle changes drastically at $t=0,4$, and $8 \mathrm{~s}$, that justifies the presence of sharp peaks in tip acceleration as well as control-torque plot at those instants. As shown in figure 15(a), peak value of the tip acceleration using collocated PD control is found as $88.50 \mathrm{~m} / \mathrm{s}^{2}$, which is reduced to $71.83 \mathrm{~m} / \mathrm{s}^{2}$ using one-mode shaped response. This means that peak vibration level of tip acceleration is reduced
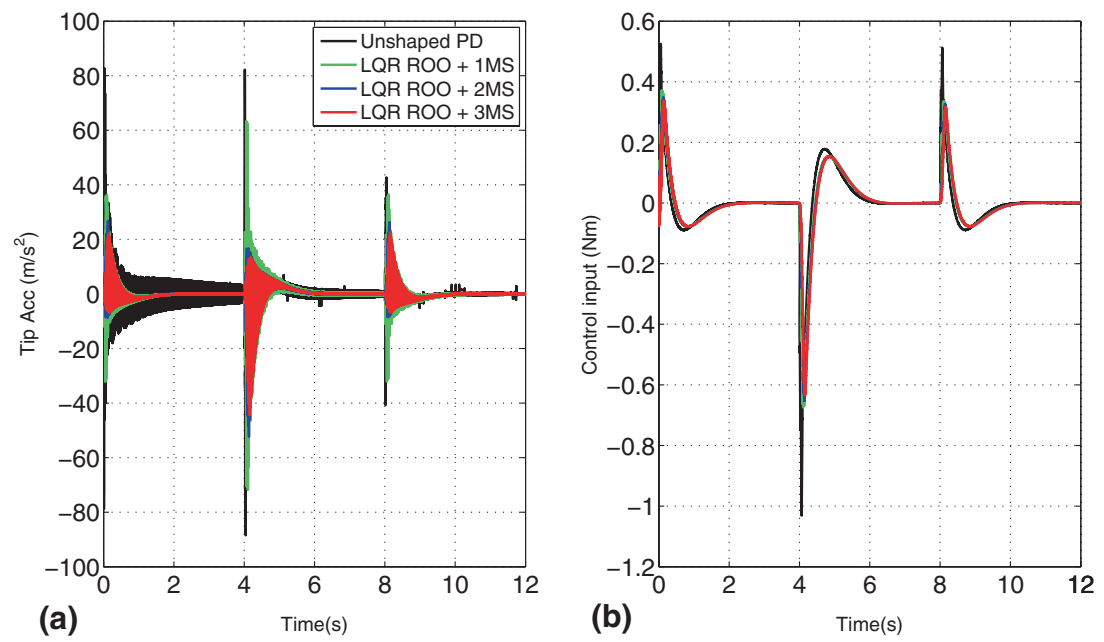

Figure 15. Comparison of unshaped vs shaped tip acceleration and control torque of a single-link flexible manipulator. 
by $18.84 \%$ by using single-mode shaped response. In the case of two-mode shaped response, the peak acceleration value is equal to $52.32 \mathrm{~m} / \mathrm{s}^{2}$, which means a reduction of $40.88 \%$. Further, using the three-mode shaped response, $44.32 \mathrm{~m} / \mathrm{s}^{2}$ is reported as the maximum tip acceleration value. The vibration level is further reduced by $9.05 \%$ as compared with the two-mode shaped response, leading to a total reduction of $49.92 \%$.

Similar trends can be observed in the control-input response, as shown in figure 15(b). Using the collocated PD control, the maximum value of the control torque is found as $1.03 \mathrm{Nm}$, which is reduced to $0.674,0.652$, and $0.632 \mathrm{Nm}$ in the case of one-mode, two-mode and three-mode shaped responses, resulting in reduction of vibration levels by $34.56 \%, 36.70 \%$, and $38.64 \%$ respectively. Both the parameters show significant reduction in vibration levels and hence validate the efficacy of the proposed control scheme in accurate tracking, reduction in vibration levels and saving the control energy of the unstable flexible manipulator.

\section{Conclusions}

The paper has investigated the performance of the hybrid controller for end-point vibration suppression of a flexible manipulator, while it is tracking a desired input profile. A hybrid controller has been used to track large hub-angle movements of flexible robotic manipulators. The hybrid controller has been developed with the combination of three different control schemes, namely inverse dynamics feedforward control, command shaping and linear state feedback control. The nonlinear feedforward control has been obtained by dynamic inversion for generating the nominal trajectory to obtain linearized models. The linear feedback has been designed with a linear quadratic regulator (LQR) which ensures stabilization as well as desired performance of the flexible systems. Further, a reduced order observer (ROO) has been designed to estimate unmeasurable states of a flexible manipulator and command shaping (CS) has been utilized to reduce the vibration levels. Both open-loop and closed-loop control schemes have been applied to demonstrate the effectiveness of the hybrid controller on a single-link flexible manipulator. In the open-loop control scheme, it has been observed that command shaping is an effective technique in reducing the structural vibrations of flexible systems. Moreover, it has been demonstrated well that ZVDD shaper is more efficient in handling payload variations as compared to a ZV shaper. Under the closed-loop control scheme, it has been shown that the hybrid controller is effective in tracking the large-hub angle trajectory of a single-link flexible manipulator, while maintaining low vibration levels. In comparison to collocated PD control, large reductions in tip acceleration levels as well as input torque magnitudes have been observed with the proposed hybrid controller.

\section{Appendix}

Element equations of motion. In this section, the equations of motion of a beam element are derived, based on the Euler-Bernoulli theory. The beam is divided into elements, where each node of the element possesses two degrees of freedom. The degrees of freedom of $i$-th node are $u_{2 i-1}$ (transverse displacement) and $u_{2 i}$ (slope). The element local displacement vector is represented as $\mathbf{u}^{e}=\left\{u_{1}, u_{2}, u_{3}, u_{4}\right\}^{T}$. The transverse displacement $y(x, t)$ within the element is given by

$$
y(x, t)=\sum_{i=1}^{4} \phi_{i}(x) u_{i}(t),
$$


where $\phi_{i}(x)$ are the shape functions which must satisfy the nodal displacement and slope continuity requirements. The prescribed boundary conditions on the beam element can be given as

$$
\begin{array}{ll}
y(0, t)=u_{1}(t), & \frac{\partial y}{\partial x}(0, t)=u_{2}(t), \\
y(l, t)=u_{3}(t), & \frac{\partial y}{\partial x}(l, t)=u_{4}(t) .
\end{array}
$$

Subject to the element boundary conditions, the shape functions must satisfy the boundary conditions, as given in table 7. As there are four constraints on each interpolation function, cubic shape functions are selected, which are of the following form

$$
\phi_{i}(x)=a_{i}+b_{i} x+c_{i} x^{2}+d_{i} x^{3}, \quad i=1, \ldots, 4 .
$$

Eliminating the constants using Eq. (80), subject to the boundary conditions given in table 7, the shape functions for the $i$-th element can be represented as

$$
\begin{aligned}
\phi(x) & =1-3 \frac{x^{2}}{l^{2}}+2 \frac{x^{3}}{l^{3}}, \\
\phi_{2}(x) & =x-2 \frac{x^{2}}{l}+\frac{x^{3}}{l^{2}}, \\
\phi_{3}(x) & =3 \frac{x^{2}}{l^{2}}-2 \frac{x^{3}}{l^{3}}, \\
\phi_{4}(x) & =-\frac{x^{2}}{l}+\frac{x^{3}}{l^{2}},
\end{aligned}
$$

which are known as Hermite polynomials (Meirovitch 1986). Substituting Eq. (81) into Eq. (78), the transverse deflection of the beam element can be expressed as

$$
\begin{aligned}
y(x, t)= & \left(1-3 \frac{x^{2}}{l^{2}}+2 \frac{x^{3}}{l^{3}}\right) u_{1}(t)+\left(\frac{x}{l}-2 \frac{x^{2}}{l^{2}}+\frac{x^{3}}{l^{3}}\right) l u_{2}(t)+ \\
& \left(3 \frac{x^{2}}{l^{2}}-2 \frac{x^{3}}{l^{3}}\right) u_{3}(t)+\left(-\frac{x^{2}}{l^{2}}+\frac{x^{3}}{l^{3}}\right) l u_{4}(t) .
\end{aligned}
$$

Further, the elemental kinetic energy can be computed as

$$
T=\frac{1}{2} \int_{0}^{l} \rho(x)[\dot{y}(x, t)]^{2} d x .
$$

Table 7. Boundary conditions on shape functions.

\begin{tabular}{ccccccccc}
\hline$x$ & $\phi$ & $\phi^{\prime}$ & $\phi_{2}$ & $\phi_{2}^{\prime}$ & $\phi_{3}$ & $\phi_{3}^{\prime}$ & $\phi_{4}$ & $\phi_{4}^{\prime}$ \\
\hline 0 & 1 & 0 & 0 & 1 & 0 & 0 & 0 & 0 \\
$l$ & 0 & 0 & 0 & 0 & 1 & 0 & 0 & 1 \\
\hline
\end{tabular}


Using Eq. (78), kinetic energy of the element can be expressed as

$$
\begin{aligned}
T & =\frac{1}{2} \int_{0}^{l} \rho(x)\left[\sum_{i=1}^{4} \phi_{i}(x) \dot{u}_{i}(t)\right]\left[\sum_{j=1}^{4} \phi_{j}(x) \dot{u}_{j}(t)\right] d x \\
& =\frac{1}{2} \sum_{i=1}^{4} \sum_{j=1}^{4} \dot{u}_{i}(t) \dot{u}_{j}(t)\left[\int_{0}^{l} \rho(x) \phi_{i}(x) \phi_{j}(x)\right] d x .
\end{aligned}
$$

From the above expression, elemental mass matrix can be computed as

$$
\begin{aligned}
\mathbf{M}^{e} & =\int_{0}^{l} \rho(x) \phi_{i}(x) \phi_{j}(x) d x \quad \forall i=j=1, \ldots, 4 \\
& =\frac{\rho l}{420}\left[\begin{array}{cccc}
156 & 22 l & 54 & -13 l \\
22 l & 4 l^{2} & 13 l & -3 l^{2} \\
54 & 13 l & 156 & -22 l \\
-13 l & -3 l^{2} & -22 l & 4 l^{2}
\end{array}\right]
\end{aligned}
$$

On the other hand, elemental potential energy can be expressed as

$$
V=\frac{1}{2} \int_{0}^{l} E I(x)\left[y^{\prime \prime}(x, t)\right]^{2} d x,
$$

which can be approximated as

$$
\begin{aligned}
V & =\frac{1}{2} \int_{0}^{l} E I(x)\left[\sum_{i=1}^{4} \phi_{i}^{\prime \prime}(x) u_{i}(t)\right]\left[\sum_{j=1}^{4} \phi_{j}^{\prime \prime}(x) u_{j}(t)\right] d x \\
& =\frac{1}{2} \sum_{i=1}^{4} \sum_{j=1}^{4} u_{i}(t) u_{j}(t)\left[\int_{0}^{l} E I(x) \phi_{i}^{\prime \prime}(x) \phi_{j}^{\prime \prime}(x)\right] d x .
\end{aligned}
$$

From the above expression, elemental stiffness matrix can be computed as

$$
\begin{aligned}
\mathbf{K}^{e} & =\int_{0}^{l} E I(x) \phi_{i}^{\prime \prime}(x) \phi_{j}^{\prime \prime}(x) \quad \forall i=j=1, \ldots, 4 \\
& =\frac{E I}{l^{3}}\left[\begin{array}{cccc}
12 & 6 l & -12 & 6 l \\
6 l & 4 l^{2} & -6 l & 2 l^{2} \\
-12 & -6 l & 12 & -6 l \\
6 l & 2 l^{2} & -6 l & 4 l^{2}
\end{array}\right] .
\end{aligned}
$$

Finally, generalized force vector is given as

$$
\begin{aligned}
\mathbf{f}^{e} & =\int_{0}^{l} f(x, t) \phi_{i}(x) d x \quad \forall i=1, \ldots, 4 \\
& =\frac{f l}{12}\left[\begin{array}{c}
6 \\
l \\
6 \\
-l
\end{array}\right]
\end{aligned}
$$

where $f(x, t)$ is the transverse load uniformly distributed over the beam element. 
Using the Lagrange equations, elemental equations of motion are given as

$$
\mathbf{M}^{e} \ddot{\mathbf{u}}^{e}+\mathbf{K}^{e} \mathbf{u}^{e}=\mathbf{f}^{e} .
$$

Assembling process. In the previous section - mass matrix, stiffness matrix and nodal force vector are developed at the elemental level. In the present section, results obtained at elemental level are extended for the complete structure as an assemblage of individual elements. The complete structure can be adequately modeled by incorporating the constraint of geometric compatibility at each element node, i.e. nodal displacements - translations and rotations, shared by several elements must be same. The nodal forces - forces and torques, must be statically equivalent to the applied forces. The common set of local displacement coordinates become the generalized coordinates of the whole structure. The whole assembly procedure has been presented below with a fixed-free beam of length $L$, which is divided into $n$ elements as shown in figure 16. The global displacement vector for the whole structure is given as $\overline{\mathbf{u}}^{g}=$ $\left\{u_{1}, u_{2}, \ldots, u_{2 n+2}\right\}^{T}$. By considering the continuity of nodal displacements, global mass matrix $\left(\overline{\mathbf{M}}^{g}\right)$ can be represented as

$$
\overline{\mathbf{M}}^{g}=\frac{\rho l}{420}\left[\begin{array}{ccccccc}
\overline{\mathbf{M}}_{11} & \overline{\mathbf{M}}_{12} & \mathbf{0} & \mathbf{0} & \ldots & \mathbf{0} & \mathbf{0} \\
\overline{\mathbf{M}}_{12}^{T} & \overline{\mathbf{M}}_{22} & \overline{\mathbf{M}}_{12} & \mathbf{0} & \ldots & \mathbf{0} & \mathbf{0} \\
\mathbf{0} & \overline{\mathbf{M}}_{12}^{T} & \overline{\mathbf{M}}_{22} & \overline{\mathbf{M}}_{12} & \ldots & \mathbf{0} & \mathbf{0} \\
\vdots & \vdots & \vdots & \vdots & \ldots & \vdots & \vdots \\
\mathbf{0} & \mathbf{0} & \mathbf{0} & \mathbf{0} & \ldots & \overline{\mathbf{M}}_{22} & \overline{\mathbf{M}}_{12} \\
\mathbf{0} & \mathbf{0} & \mathbf{0} & \mathbf{0} & \ldots & \overline{\mathbf{M}}_{12}^{T} & \overline{\mathbf{M}}_{33}
\end{array}\right]
$$

where

$$
\begin{array}{ll}
\overline{\mathbf{M}}_{11}=\left[\begin{array}{cc}
156 & 22 l \\
22 l & 4 l^{2}
\end{array}\right], & \overline{\mathbf{M}}_{12}=\left[\begin{array}{cc}
54 & -13 l \\
13 l & -3 l^{2}
\end{array}\right], \\
\overline{\mathbf{M}}_{22}=\left[\begin{array}{cc}
312 & 0 \\
0 & 8 l^{2}
\end{array}\right], & \overline{\mathbf{M}}_{33}=\left[\begin{array}{cc}
156 & -22 l \\
-22 l & 4 l^{2}
\end{array}\right] .
\end{array}
$$

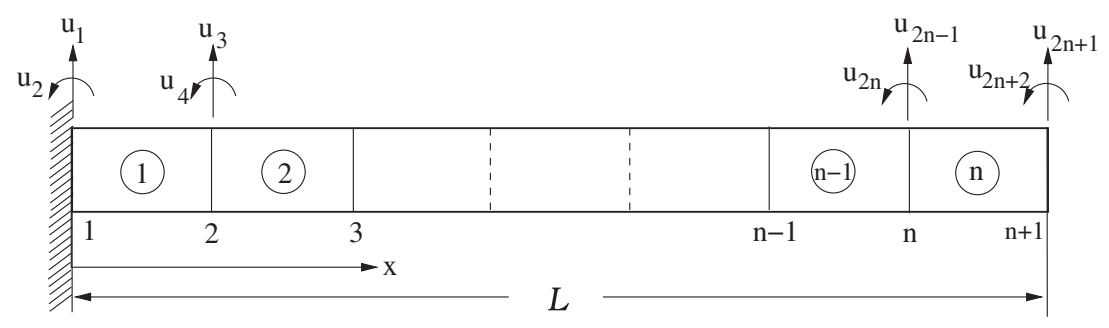

Figure 16. A fixed-free beam discretized into $n$ elements. 
Following a similar process, global stiffness matrix $\left(\overline{\mathbf{K}}^{g}\right)$ is given as

$$
\overline{\mathbf{K}}^{g}=\frac{E I}{l^{3}}\left[\begin{array}{ccccccc}
\overline{\mathbf{K}}_{11} & \overline{\mathbf{K}}_{12} & \mathbf{0} & \mathbf{0} & \ldots & \mathbf{0} & \mathbf{0} \\
\overline{\mathbf{K}}_{12}^{T} & \overline{\mathbf{K}}_{22} & \overline{\mathbf{K}}_{12} & \mathbf{0} & \ldots & \mathbf{0} & \mathbf{0} \\
\mathbf{0} & \overline{\mathbf{K}}_{12}^{T} & \overline{\mathbf{K}}_{22} & \overline{\mathbf{K}}_{12} & \ldots & \mathbf{0} & \mathbf{0} \\
\vdots & \vdots & \vdots & \vdots & \ldots & \vdots & \vdots \\
\mathbf{0} & \mathbf{0} & \mathbf{0} & \mathbf{0} & \ldots & \overline{\mathbf{K}}_{22} & \overline{\mathbf{K}}_{12} \\
\mathbf{0} & \mathbf{0} & \mathbf{0} & \mathbf{0} & \ldots & \overline{\mathbf{K}}_{12}^{T} & \overline{\mathbf{K}}_{33}
\end{array}\right]
$$

where

$$
\begin{array}{ll}
\overline{\mathbf{K}}_{11}=\left[\begin{array}{cc}
12 & 6 l \\
6 l & 4 l^{2}
\end{array}\right], & \overline{\mathbf{K}}_{12}=\left[\begin{array}{cc}
-12 & 6 l \\
-6 l & 2 l^{2}
\end{array}\right], \\
\overline{\mathbf{K}}_{22}=\left[\begin{array}{cc}
24 & 0 \\
0 & 8 l^{2}
\end{array}\right], & \overline{\mathbf{K}}_{33}=\left[\begin{array}{cc}
12 & -6 l \\
-6 l & 4 l^{2}
\end{array}\right] .
\end{array}
$$

It is worth mentioning that both global mass and stiffness matrices are of size $(2 n+2) \times$ $(2 n+2)$ and are symmetric in nature.

Boundary conditions. The whole assembly procedure is presented in the previous section, where each element node is assumed to be unrestrained. This implies that all nodes of the structure can undergo displacements i.e. the whole structure can undergo rigid body motion under the joint forces, which leads to a singular stiffness matrix. However, structures are supported in general to prevent the rigid body mode. Thus, it is necessary to incorporate the boundary conditions in equations of motion of the complete structure. Boundary condition are generally of two types - essential boundary conditions (prescribed displacements) and natural boundary conditions (prescribed forces). While modeling the structure with FEM, force boundary condition are taken into account while evaluating the externally applied nodal force vector. However, displacement boundary conditions are imposed on the global matrices. For example, in the case of clamped-free beam discussed above, left-end of the beam is fixed, which leads to the following boundary conditions

$$
\begin{gathered}
y(x=0, t)=0 \Rightarrow u_{1}(t)=0, \\
\frac{\partial y}{\partial x}(x=0, t)=0 \Rightarrow u_{2}(t)=0 .
\end{gathered}
$$

These boundary conditions are implemented by deleting the first two rows and columns of global mass and stiffness matrices. In addition, force distribution on the beam is assumed to be zero i.e. $f(x, t)=0$. The modified global displacement vector for the whole structure is of size $2 n \times 1$ and can be given as $\mathbf{u}^{g}=\left\{u_{3}, u_{4}, \ldots, u_{2 n+2}\right\}^{T}$. Referring back to Eqs. (91) and 
(93), after imposing the boundary conditions, global mass and stiffness matrices can be modified as

$$
\mathbf{M}^{g}=\frac{\rho l}{420}\left[\begin{array}{ccccccc}
\mathbf{M}_{11} & \mathbf{M}_{12} & \mathbf{0} & \mathbf{0} & \ldots & \mathbf{0} & \mathbf{0} \\
\mathbf{M}_{12}^{T} & \mathbf{M}_{11} & \mathbf{M}_{12} & \mathbf{0} & \ldots & \mathbf{0} & \mathbf{0} \\
\mathbf{0} & \mathbf{M}_{12}^{T} & \mathbf{M}_{11} & \mathbf{M}_{12} & \ldots & \mathbf{0} & \mathbf{0} \\
\vdots & \vdots & \vdots & \vdots & \ldots & \vdots & \vdots \\
\mathbf{0} & \mathbf{0} & \mathbf{0} & \mathbf{0} & \ldots & \mathbf{M}_{11} & \mathbf{M}_{12} \\
\mathbf{0} & \mathbf{0} & \mathbf{0} & \mathbf{0} & \ldots & \mathbf{M}_{12}^{T} & \mathbf{M}_{22}
\end{array}\right]
$$

where

$$
\begin{gathered}
\mathbf{M}_{11}=\overline{\mathbf{M}}_{22}, \\
\mathbf{K}^{g}=\frac{E I}{l^{3}}\left[\begin{array}{cccccccc}
\mathbf{K}_{11} & \mathbf{K}_{12} & \mathbf{0} & \mathbf{0} & \ldots & \mathbf{0} & \mathbf{0} \\
\mathbf{K}_{12}^{T} & \mathbf{K}_{11} & \mathbf{K}_{12} & \mathbf{0} & \ldots & \mathbf{0} & \mathbf{0} \\
\mathbf{0} & \mathbf{K}_{12}^{T} & \mathbf{K}_{11} & \mathbf{K}_{12} & \ldots & \mathbf{0} & \mathbf{0} \\
\vdots & \vdots & \vdots & \vdots & \ldots & \vdots & \vdots \\
\mathbf{0} & \mathbf{0} & \mathbf{0} & \mathbf{0} & \ldots & \mathbf{K}_{11} & \mathbf{K}_{12} \\
\mathbf{0} & \mathbf{0} & \mathbf{0} & \mathbf{0} & \ldots & \mathbf{K}_{12}^{T} & \mathbf{K}_{22}
\end{array}\right],
\end{gathered}
$$

where

$$
\mathbf{K}_{11}=\overline{\mathbf{K}}_{22}, \quad \mathbf{K}_{22}=\overline{\mathbf{K}}_{33}, \quad \mathbf{K}_{12}=\overline{\mathbf{K}}_{12} .
$$

After imposing the boundary conditions, dimension of the system $\left[\mathbf{M}^{g}, \mathbf{K}^{g}, \mathbf{u}^{g}\right]$ is reduced to $2 n$. Finally, using the Lagrange equations, equations of motion for the whole structure are given in the form

$$
\mathbf{M}^{g} \ddot{\mathbf{u}}^{g}+\mathbf{K}^{g} \mathbf{u}^{g}=\mathbf{0} .
$$

\section{References}

Banerjee A and Singhose W 1998 Command shaping in tracking control of a two-link flexible robot. $J$. Guidance Control Dyn. 21(6): 1012-1015

Book W J and Majette M 1983 Controller design for flexible, distributed parameter mechanical arms via combined state space and frequency domain techniques. J. Dyn. Syst. Measur. Control 105(4): 245-254 
Cambera J C, Chocoteco J A and Feliu V 2014 Feedback linearizing controller for a flexible single-link arm under gravity and joint friction. In: ROBOT2013: First Iberian Robotics Conference, pages 169-184. Springer

De Luca A and Siciliano B 1991 Closed-form dynamic model of planar multilink lightweight robots. IEEE Trans. Syst. Man Cybern. 21(4): 826-839

Drapeau V and Wang D 1993 Verification of a closed-loop shaped-input controller for a five-bar-linkage manipulator. IEEE International Conference on Robotics and Automation, pages 216-221

Feliu V, Rattan K S and Brown H B 1990 Adaptive control of a single-link flexible manipulator. IEEE Control Syst. Mag. 10(2): 29-33

Hillsley K L and Yurkovich S 1993 Vibration control of a two-link flexible robot arm. Dyn. Control 3(3): 261-280

Hyde J M and Seering W P 1991 Using input command pre-shaping to suppress multiple mode vibration. IEEE International Conference on Robotics and Automation, pages 2604-2609

Junkins J L and Kim Y 1993 Introduction to dynamics and control of flexible structures. Aiaa

Kapila V, Tzes A and Yan Q 2000 Closed-loop input shaping for flexible structures using time-delay control. J. Dyn. Syst. Measur. Control 122: 454-460

Kenison M and Singhose W 2002 Concurrent design of input shaping and proportional plus derivative feedback control. J. Dyn. Syst. Measur. Control 124: 398-405

Meirovitch L 1986 Elements of vibrational analysis. New York: McGraw-Hill

Mohamed Z and Tokhi M O 2002 Vibration control of a single-link flexible manipulator using command shaping techniques. Proc. Inst. Mech. Eng. Part I: J. Syst. Control Eng. 216(2): 191-210

Mohan A and Saha S 2009 A recursive, numerically stable, and efficient simulation algorithm for serial robots with flexible links. Multibody Syst. Dyn. 21(1): 1-35

Peláez G, Pelaez G, Perez J, Vizán A and Bautista E 2005 Input shaping reference commands for trajectory following Cartesian machines. Control Eng. Practice 13(8): 941-958

Rattan K S and Feliu V 1991 A robust control scheme for flexible manipulators. IEEE National aerospace and electronics conference 1090-1095

Romano M, Agrawal B N and Bernelli-Zazzera F 2002 Experiments on command shaping control of a manipulator with flexible links. J. Guidance Control Dyn. 25(2): 232-239

Shaheed M H and Tokhi O 2013 Adaptive closed-loop control of a single-link flexible manipulator. J. Vibr. Control 19(13): 2068-2080

Singer N C and Seering W P 1990 Preshaping command inputs to reduce system vibration. J. Dyn. Syst. Measur. Control Trans. ASME 112(1): 76-82

Singh T and Vadali S R 1994 Robust time-optimal control: Frequency domain approach. J. Guidance Control Dyn. 17(2): 346-353

Singhose W, Derezinski S and Singer N 1996 Extra-insensitive input shapers for controlling flexible spacecraft. J. Guidance Control Dyn. 19(2): 385-391

Tewari A 2002 Modern control design with MATLAB and SIMULINK. Chichester: Wiley

Tewari A 2004 Active vibration suppression of spacecraft with rate-weighted optimal control and multimode command shaping. In: AIAA/AAS astrodynamics specialist conference and exhibit, providence, Rhode Island

Tokhi M O, Mohamed Z and Shaheed M H 2001 Dynamic characterisation of a flexible manipulator system. Robotica 19(5): 571-580

Tzes A and Yurkovich S 1993 An adaptive input shaping control scheme for vibration suppression in slewing flexible structures. IEEE Trans. Control Syst. Technol. 1(2): 114-121

Tzou H S 1989 Nonlinear structural dynamics of space manipulators with elastic joints. Int. J. Anal. Exp. Modal Anal. 4: 117-123

Usoro P B, Mahil S S and Nadira R 1984 A finite element/Lagrange approach to modeling lightweight flexible manipulators, part one: one-link system. Sensors and Controls for Automated Manufacturing and Robotics $215-228$

Usoro P B, Nadira R and Mahil S S 1986 A finite element/Lagrange approach to modeling lightweight flexible manipulators. J. Dyn. Syst. Measur. Control 108: 198-205 
Vaughan J, Yano A and Singhose W 2008 Comparison of robust input shapers. J. Sound Vibr. 315: 797-815 Yigit A. S. 1994 On the Stability of PD Control for a Two-Link Rigid-Flexible Manipulator. J. Dyn. Syst. Measur. Control 116: 179-185

Zain M, Tokhi M O and Mohamed Z 2006a Hybrid learning control schemes with input shaping of a flexible manipulator system. Mechatronics 16(3-4): 209-219

Zain M, Tokhi M O and Mohamed Z 2006b Performance of hybrid learning control with input shaping for input tracking and vibration suppression of a flexible manipulator. J. Teknologi 44(A): 41-64

Zhang N, Feng Y and Yu X 2004 Optimization of terminal sliding control for two-link flexible manipulators. IEEE 30th annual conference of industrial electronics society 2: 1318-1322 